Pure and Applied Mathematics Quarterly

Volume 5, Number 1

(Special Issue: In honor of

Jean-Pierre Serre, Part 2 of 2)

$507-570,2009$

\title{
Zeta Functions and Motives
}

\author{
Bruno Kahn
}

\section{À Jean-Pierre Serre}

\begin{abstract}
We study properties of rigid $K$-linear $\otimes$-categories $\mathcal{A}$, where $K$ is a field of characteristic 0 . When $\mathcal{A}$ is semi-simple, we introduce a notion of multiplicities for an object of $\mathcal{A}$ : they are rational integers in important cases including that of pure numerical motives over a field. This yields an alternative proof of the rationality and functional equation of the zeta function of an endomorphism, and a simple proof that the number of rational points modulo $q$ of a smooth projective variety over $\mathbf{F}_{q}$ only depends on its "birational motive". The multiplicities of motives of abelian type over a finite field are equal to \pm 1 . We also study motivic zeta functions, and an abstracted version of the Tate conjecture over finite fields.
\end{abstract}

Keywords: Zeta functions, motives, finite-dimensional motives, Tate conjecture, Grothendieck groups of additive categories.

This article consists of four independent but related parts, whose common themes are motives and zeta functions. Another important theme is the Tate conjecture.

In Part I, I introduce the notion of multiplicity of a numerical motive, or more generally of an object in a semi-simple rigid $K$-linear tensor category where $K$ is a field of characteristic 0 . In the case of motives, or more generally under a Schurfiniteness assumption, these multiplicities are rational integers. One application is a proof "without cohomology" of the rationality and functional equation for

Received February 12, 2008. 
the zeta function of an endomorphism in such a category (see Theorem 3.2). The main result of Part I, Theorem 6.3, is that the multiplicities of a motive of abelian type over a finite field are all equal to \pm 1 (recall that conjecturally all motives over a finite field are of abelian type). This is a revised version of a preprint of February 2007 [34].

In Part II, the zeta functions are in the background. The main result is that the number of points modulo $q$ of a smooth projective variety $X$ over $\mathbf{F}_{q}$ only depends on the birational motive of $X$ in the sense of [36]. This is a revised version of a 2002 preprint [31]; the main novelty is a simplified proof of the latter fact, using the multiplicities of Part I (in fact, this application was my initial motivation for studying multiplicities). I also point out in Remark 10.32 ) that, thanks to Honda's theorem, the Tate conjecture over a finite field implies the generalised Tate conjecture, an observation which was only implicit in [31, proof of Th. 2], cf. [2, p. 81 fn. 9]. (This has since been observed independently by Milne and Ramachandran, see [49, §1]).

In Part III, I prove that the motivic zeta function of a (pure) motive over a field $k$ which is finite-dimensional in the sense of Kimura-O'Sullivan satisfies a functional equation. As in Part I, the result is more general and applies to zeta functions associated to finite-dimensional objects of any rigid tensor category such that the endomorphism ring of the unit object is a field of characteristic zero. This is a slightly revised version of a preprint of June 2006 [33]: the main differences are a more functorial theory of the determinant (see Proposition 11.3) which leads to a (slightly) more conceptual understanding of the functional equation, and an extra section relating motivic zeta functions with the zeta functions of endomorphisms from $\S 3$.

Part IV is extracted from the first version of Part I: I found more convenient to put it separately. It shows that the arguments of Tate in [57], Geisser in [24] and myself in [32] concerning the relationships between the conjectures of Tate and Beilinson $^{1}$ and some of the standard conjectures for smooth projective varieties over a finite field are in fact abstract in nature and may be formulated in a rather

\footnotetext{
${ }^{1}$ In $[48, \S 1]$, Milne points out that Beilinson's conjecture that rational and numerical equivalence coincide over a finite field was already mentioned by Tate in the Woods-Hole 1964 AMS Summer Institute, where he introduced his conjecture.
} 
general semi-simple tensor category. The multiplicities of Part I are important for the proof of Proposition 17.6.

I had planned to add a part concerning motivic zeta functions of Voevodsky motives over a base, but this raised unexpected issues. I hope to come back to this question later.

Each part has its own expanded introduction.

Acknowledgements. Concerning Parts I and IV I thank Yves André, Alessio del Padrone and Chuck Weibel for helpful exchanges during its preparation. Part of this research was done during a visit to the Tata Institute of Fundamental Research in November 2006, partially funded by the Indo-French Institute for Mathematics: I would like to thank both institutions for their hospitality and support. Part of the work in Part II was done during a stay at the Newton Institute of Mathematical Sciences in September-October 2002; I thank it for its hospitality. I am grateful to Dan Abramovich, Yves André, Antoine ChambertLoir, Jean-Louis Colliot-Thélène, Najmuddin Fakhruddin, Jean-Marc Fontaine, Ofer Gabber, Uwe Jannsen, Gilles Lachaud and Yves Laszlo for helpful remarks. Especially André helped with the initial proof of Theorem 8.1, Colliot-Thélène and Chambert-Loir pointed out the work of Lachaud and Perret [45], ChambertLoir pointed out the work of Ekedahl [18] and Lachaud kindly communicated the letters of Serre [54]. The idea of this work was of course sparked by Hélène Esnault's article [19]. Concerning Part III, I wish to thank Noriyuki Otsubo for an enlightening discussion, Yves André for several comments on its first draft, Jean-Louis Colliot-Thélène for his help in the example of Remark 14.6 c), and Marc Hindry and Georges Skandalis for their help in proving the identity in the proof of Proposition 15.5. Finally, thanks are due to the referees whose sharp observations helped me to improve the exposition, and actually the mathematics of this paper!

\section{Contents}

Acknowledgements 
Terminology and notation

1. Multiplicities in semi-simple rigid tensor categories 513

2. Integral multiplicities $\quad 515$

3. Application: the zeta function of an endomorphism 518

4. Objects of constant sign and finite dimensionality 519

5. Multiplicities in rigid tensor categories of homological type 521

6. Examples 526

Part II. Number of points of function fields over finite fields $\quad 528$

Introduction $\quad 528$

$\begin{array}{ll}\text { 7. Birational motives } & 529\end{array}$

8. An integrality theorem 532

9. Number of rational points modulo $q$

10. A conjectural converse 536

Part III. Motivic zeta functions of motives 537

$\begin{array}{ll}\text { Introduction } & 537\end{array}$

11. The determinant 539

12. The functional equation 543

13. The case of motives $\quad 545$

14. The determinant of a motive 548

15. An extension: zeta functions of endomorphisms 552

Part IV. An abstract version of the Tate(-Beilinson) conjecture 555

Introduction $\quad 555$

16. Automorphisms of the identity functor 555

17. The semi-simple case 557 
19. The Tate-Beilinson conjecture 566

$\begin{array}{ll}\text { References } & 567\end{array}$

\section{Part I. On the multiplicities of a motive}

\section{INTRODUCTION}

The aim of this part and Part IV, in the spirit of [4], is to study abstractly the properties of categories of pure motives and to make clear(er) which of them are formal and which are of a more arithmetic-geometric nature.

We work with a rigid additive tensor category $\mathcal{A}$ such that $K=\operatorname{End}(\mathbf{1})$ is a field of characteristic 0 . We shall be interested in the multiplicities of an object $M \in \mathcal{A}$ : when $\mathcal{A}$ is semi-simple, they are a collection of central scalars which relates the categorical trace with the ring-theoretic trace (Proposition 1.2). It turns out that the condition for these multiplicities to be integers or, better, to be so after extending scalars from $K$ to its algebraic closure, is well-behaved and satisfied in many important cases.

Namely, the full subcategory $\mathcal{A}_{\text {int }}$ of $\mathcal{A}$ formed by such objects is stable under direct sums, direct summands and duals and contains the "Schur-finite" objects (those which are killed by a nonzero Schur functor). As a consequence, $\mathcal{A}_{\text {int }}=\mathcal{A}$ if $\mathcal{A}$ is of "homological origin" (Theorem 5.6).

As a main motivational example, recall that the category of pure motives over a field modulo numerical equivalence is semi-simple thanks to Jannsen's theorem [29], and of homological origin: thus, the multiplicities of any pure motive modulo numerical equivalence are integers.

When the multiplicities of $M \in \mathcal{A}$ are integers, we prove that the zeta function of an endomorphism $f$ of $M$ is rational (with an explicit formula) and satisfies a functional equation if $f$ is invertible (Theorem 3.2): in the case of motives over a finite field, this shows that these properties depend on less than the existence of a Weil cohomology theory. 
In a general $\mathcal{A}$, it is not necessarily true that the tensor product of two geometrically integral objects $M, N$ is geometrically integral (see Remark 2.5). However, when this happens to be the case (e.g. when $M$ and $N$ are Schur-finite), there are important explicit formulas allowing one to compute the multiplicities of $M \otimes N$ in terms of those of $M$ and $N$ (Theorem 2.4). We use this to get some elementary cases where homological equivalence equals numerical equivalence for formal reasons in Proposition $5.10 \mathrm{c}$ ): of course, this remains far from leading to a proof of this famous standard conjecture!

Finally, we compute the multiplicities of numerical motives of abelian type over a finite field and show that they are all equal to \pm 1 (Theorem 6.3).

Terminology and notation. For a category $\mathcal{C}$ and two objects $X, Y \in \mathcal{C}$, we write indifferently $\operatorname{Hom}_{\mathcal{C}}(X, Y)$ or $\mathcal{C}(X, Y)$ for the set of morphisms from $X$ to $Y$ (the latter notation is convenient when the name of $\mathcal{C}$ is large).

Let $\mathcal{A}$ be a rigid $K$-linear symmetric monoidal (=tensor) category, where $K$ is a field of characteristic 0 ; we also assume that $\operatorname{End}(\mathbf{1})=K$. In the sequel of this article, we shall abbreviate this by saying that $\mathcal{A}$ is a rigid $K$-category. Since we shall refer to Deligne's article [13] several times, it is worth stressing that we do not assume $\mathcal{A}$ abelian, unlike in loc. cit. Recall that $\mathcal{A}$ is called pseudo-abelian (or idempotent-closed) if any idempotent endomorphism has a kernel (equivalently, an image). We write $\mathcal{A}^{\natural}$ for the pseudo-abelian hull (idempotent completion) of $\mathcal{A}[4,1.2 .2]$. If $L$ is an extension of $K$, we write $\mathcal{A}_{L}$ for the category with the same objects and Hom groups tensored with $L$.

If $M \in \mathcal{A}^{\natural}$, we shall say (as has become common practice) that $M$ is Schurfinite if there exists a nonzero Schur functor $\mathbb{S}$ such that $\mathbb{S}(M)=0$ and finitedimensional (in the sense of Kimura-O'Sullivan) if $M \simeq M^{+} \oplus M^{-}$where $M^{+}$(resp. $\left.M^{-}\right)$is killed by some nonzero exterior (resp. symmetric) power functor. We say that $M^{+}$is positive and $M^{-}$is negative. It is known that finite-dimensional implies Schur-finite $(c f$. $[13,1.13])$. For properties of finitedimensional objects (resp. of Schur functors) we refer to [39] and [4, §9] (resp. to $[13])$. 


\section{Multiplicities in SEMI-Simple Rigid tensor CATEGORIES}

Let $M \in \mathcal{A}$. The trace of an endomorphism $f \in \operatorname{End}(M)$ is the element $\operatorname{tr}(f) \in \operatorname{End}(\mathbf{1})=K$ defined by the composition

$$
\mathbf{1} \stackrel{\eta}{\longrightarrow} M^{*} \otimes M \stackrel{1 \otimes f}{\longrightarrow} M^{*} \otimes M \stackrel{R}{\longrightarrow} M \otimes M^{*} \stackrel{\varepsilon}{\longrightarrow} \mathbf{1}
$$

where $R$ is the switch and $\eta, \varepsilon$ are the duality structures of $M$.

Special case. We shall denote the trace of $1_{M}$ by $\chi(M)$ and call it the Euler characteristic of $M$.

The trace is $K$-linear and has the following properties:

$$
\operatorname{tr}(f g)=\operatorname{tr}(g f), \quad \operatorname{tr}(f \otimes g)=\operatorname{tr}(f) \operatorname{tr}(g), \quad \operatorname{tr}\left({ }^{t} f\right)=\operatorname{tr}(f) .
$$

We shall use several times the ideal $\mathcal{N}$ of morphisms universally of trace 0 :

$$
\mathcal{N}(M, N)=\{f \in \mathcal{A}(M, N) \mid \forall g \in \mathcal{A}(N, M), \operatorname{tr}(g f)=0\} .
$$

Suppose $\mathcal{A}$ is semi-simple: by [4, Th. A.2.10 (7)], this amounts to requiring that $\operatorname{End}_{\mathcal{A}}(M)$ is a semi-simple $K$-algebra for all $M$. Then $\operatorname{End}_{\mathcal{A}}(M)$ has its own trace, and we want to compare it with the categorical trace. We normalise conventions as follows:

1.1. Definition. a) Let $A$ be a finite-dimensional simple $K$-algebra. We write:

- $Z(A)$ for the centre of $A$;

- $\delta(A)=[Z(A): K]$

- $d(A)=[A: Z(A)]^{1 / 2}$.

We define the reduced trace of $A$ as

$$
\operatorname{Trd}_{A}=\operatorname{Tr}_{Z(A) / K} \circ \operatorname{Trd}_{A / Z(A)} .
$$

If $A=\prod A_{i}$ is semi-simple, with simple components $A_{i}$, we define $\operatorname{Trd}_{A}:=$ $\sum_{i} \operatorname{Trd}_{A_{i}}$.

b) If $A=\operatorname{End}_{\mathcal{A}}(M)$, we set

- $Z_{i}(M)=Z\left(A_{i}\right)$;

- $\delta_{i}(M)=\delta\left(A_{i}\right)$;

- $d_{i}(M)=d\left(A_{i}\right)$;

- $\operatorname{Trd}_{M}=\operatorname{Trd}_{A}$. 
Note that, if $L$ is an extension of $K$, then $\operatorname{Trd}_{A \otimes_{K} L}(a \otimes 1)=\operatorname{Trd}_{A}(a)$ (viewed in $L$ ) for any $a \in A$.

1.2. Proposition. There exists a unique element $\mu(M) \in \operatorname{End}(M)$ such that

$$
\operatorname{tr}(f)=\operatorname{Trd}_{M}(\mu(M) f)
$$

for any $f \in \operatorname{End}(M)$. Moreover, $\mu(M)$ is central and invertible. Hence, if $\left(e_{i}\right)$ denotes the set of central idempotents of $A=\operatorname{End}(M)$ corresponding to its simple factors $A_{i}$, we may write

$$
\mu(M)=\sum_{i} \mu_{i}(M) e_{i}
$$

with $\mu_{i}(M) \in Z_{i}(M)^{*}$.

Proof. Since $\operatorname{End}(M)$ is semi-simple, $(f, g) \mapsto \operatorname{Trd}_{M}(f g)$ is nondegenerate, which proves the existence and uniqueness of $\mu(M)$. Moreover,

$$
\operatorname{Trd}_{M}(\mu(M) f g)=\operatorname{tr}(f g)=\operatorname{tr}(g f)=\operatorname{Trd}_{M}(\mu(M) g f)=\operatorname{Trd}_{M}(f \mu(M) g)
$$

and the non-degeneracy also yields the centrality of $\mu(M)$. This element is invertible because the ideal $\mathcal{N}$ of $(1.2)$ is 0 , since $\mathcal{A}$ is semi-simple $[4,7.1 .7]$. The last assertion is obvious.

1.3. Lemma. a) We have $\mu\left(M^{*}\right)={ }^{t} \mu(M)$.

b) Suppose that $K$ is algebraically closed and $M$ is simple. Then $\mu(M)=\chi(M)$.

Proof. a) follows easily from (1.1) and the fact that the transposition induces an anti-isomorphism from $\operatorname{End}(M)$ onto $\operatorname{End}\left(M^{*}\right)$. b) is obvious, since then $\operatorname{End}(M)=K$ (recall that, by definition, $\left.\chi(M)=\operatorname{tr}\left(1_{M}\right)\right)$.

1.4. Remark. If $\mathcal{A}$ is pseudo-abelian (hence abelian, [29, Lemma 2]), the idempotents $e_{i}$ of Proposition 1.2 yield the decomposition $M=\bigoplus M_{i}$ of $M$ into its isotypical components. In particular, $\mu\left(M^{\oplus n}\right)=\mu(M)$ for any $n \geq 1$.

On the other hand, it is difficult to relate $\mu\left(M_{1}\right), \mu\left(M_{2}\right)$ and $\mu\left(M_{1} \otimes M_{2}\right)$ in general because it is difficult to say something of the map $\operatorname{End}\left(M_{1}\right) \otimes_{K} \operatorname{End}\left(M_{2}\right) \rightarrow$ $\operatorname{End}\left(M_{1} \otimes M_{2}\right)$ : it need not even be true that such a homomorphism sends the centre to the centre. For the same reason, it is difficult to state general facts on the behaviour of the invariant $\mu$ under tensor functors. We shall see that the situation improves considerably in the case of geometrically integral type, discussed in the next section. 


\section{INTEGRAL MULTIPLICITIES}

In all this section, $\mathcal{A}$ is a semi-simple rigid $K$-category.

2.1. Definition. a) An object $M \in \mathcal{A}$ is of integral type if the scalars $\mu_{i}(M)$ of Proposition 1.2 belong to $\mathbf{Z}$.

b) $M$ is geometrically of integral type if $M_{\bar{K}} \in \mathcal{A}_{\bar{K}}$ is of integral type, where $\bar{K}$ is an algebraic closure of $K$.

c) $\mathcal{A}$ is of integral type (resp. geometrically of integral type) if every $M \in \mathcal{A}$ is of integral type (resp. geometrically of integral type).

We denote by $\mathcal{A}_{\text {int }}$ the full subcategory of $\mathcal{A}$ consisting of geometrically integral objects.

2.2. Proposition. a) If $M$ is of integral type, we have

$$
\mu_{i}(M)=\frac{\operatorname{tr}\left(e_{i}\right)}{\delta_{i}(M) d_{i}(M)}
$$

for any $i$, where the notation is as in Definition 1.1 and Proposition 1.2.

b) Direct sums and direct summands of objects of integral type are of integral type. Similarly for geometrically of integral type. In particular, $\mathcal{A}$ is of integral type (resp. geometrically of integral type) if and only if its pseudo-abelian envelope is. c) If $M$ is geometrically of integral type, then it is of integral type. Moreover, if this is the case, the invariants $\mu_{i}(M)$ are "geometric" in the sense that if $L / K$ is any extension, then $\mu_{i}(M)=\mu_{i, \alpha}\left(M_{L}\right)$ for any simple factor $A_{i, \alpha}$ of $A_{i} \otimes_{K} L$.

d) $M \in \mathcal{A}$ is geometrically of integral type if and only if, in $\mathcal{A}_{\bar{K}}^{\natural}$, the Euler characteristic of every simple summand of $M_{\bar{K}}$ is an integer.

e) If $M$ is Schur-finite, it is geometrically of integral type.

f) If $M$ is (geometrically) of integral type, so is $M^{*}$.

Proof. a) and b) are obvious. For c), we have the decomposition

$$
Z_{i}(M) \otimes_{K} \bar{K} \stackrel{\sim}{\longrightarrow} \prod_{\alpha} \bar{K}
$$

where $\alpha$ runs through the distinct $K$-embeddings of $Z_{i}(M)$ into $\bar{K}$. Correspondingly, $A_{i} \otimes_{K} \bar{K}$ decomposes as a direct product

$$
A_{i} \otimes_{K} \bar{K} \simeq \prod_{\alpha} A_{i, \alpha}
$$


with $A_{i, \alpha}$ simple over $\bar{K}$. This gives a decomposition

$$
e_{i} \otimes_{K} 1=\sum_{\alpha} e_{i, \alpha}
$$

into central idempotents. But clearly, $\mu\left(M_{\bar{K}}\right)=\mu(M) \otimes_{K} 1$. By hypothesis, the images of $\mu_{i}(M)$ in $\bar{K}$ under the embeddings $\alpha$ are rational integers, which implies that $\mu_{i}(M)$ is itself a rational integer. The additional claim of c) immediately follows from this proof.

d) follows immediately from Lemma $1.3 \mathrm{~b}$ ).

For e), if $M$ is Schur-finite, so is $M_{\bar{K}} \in \mathcal{A}_{\bar{K}}^{\natural}$; all simple direct summands of $M_{\bar{K}}$ are Schur-finite as well, hence their Euler characteristics are rational integers. This immediately follows from the main result of [13], but one can more elementarily use Proposition 2.2.2 of del Padrone's thesis [15], which generalises the case of finite-dimensional objects $[4,7.2 .4$ and 9.1.7]. The conclusion now follows from d).

Finally, f) follows from Lemma 1.3 a).

2.3. Remark. C. Weibel asked whether the converse of e) is true. This holds if $\mathcal{A}_{\text {int }}$ is of homological origin in the sense of Definition 5.1 b) (see Theorem 5.6), but is false in general as observed by A. del Padrone [16, (4)].

2.4. Theorem. Let $M, N \in \mathcal{A}$ be geometrically of integral type and such that $M \otimes N$ is geometrically of integral type. Let $\left(e_{i}\right)$ be the central idempotents of $\operatorname{End}(M)$ and $\left(f_{j}\right)$ the central idempotents of $\operatorname{End}(N)$. For a pair $(i, j)$, let $A_{i j}$ be the semi-simple algebra $\left(e_{i} \otimes f_{j}\right) \operatorname{End}(M \otimes N)\left(e_{i} \otimes f_{j}\right)$. Then one has formulas of the type

$$
\mu_{i}(M) \mu_{j}(N)=\sum_{k} m_{k} \mu_{k}(M \otimes N)
$$

where $k$ indexes the simple factors of $A_{i j}$ and the $m_{k}$ are integers $\geq 0$. Moreover, for any $k$, there is such a formula with $m_{k}>0$.

Proof. We proceed in 3 steps:

1) $\operatorname{End}(M)$ and $\operatorname{End}(N)$ are split and $M$ and $N$ are simple. Then $\operatorname{End}(M)=$ $\operatorname{End}(N)=K$ and $A_{i j}=\operatorname{End}(M \otimes N)$. Using Formula (2.1) to compute $\operatorname{tr}\left(1_{M} \otimes\right.$ $\left.1_{N}\right)$ in two different ways, we get the formula

$$
\mu(M) \mu(N)=\sum m_{k} \mu_{k}(M \otimes N)
$$


with $m_{k}=\delta_{k}(M \otimes N) d_{k}(M \otimes N)$.

2) $\operatorname{End}(M)$ and $\operatorname{End}(N)$ are split, which means that their centres are products of copies of $K$ and all their simple factors are matrix algebras over $K$. By Proposition $2.2 \mathrm{~b}$ ), we may assume that $\mathcal{A}$ is pseudo-abelian, and we are reduced to 1) by Remark 1.4.

Note that, as a by-product, we get the formula

$$
m_{k}=\delta_{k}\left(A_{i j}\right) \frac{d_{k}\left(A_{i j}\right)}{d_{i}(M) d_{j}(N)}
$$

and 1) shows ungrievously that the right hand side is an integer.

3) The general case. Extending scalars to $\bar{K}$ and using Proposition 2.2 c), we are reduced to 2) as follows: for any $\alpha: Z_{i}(M) \rightarrow \bar{K}$ and any $\beta: Z_{j}(M) \rightarrow \bar{K}$, we have a formula with obvious notation:

$$
\mu_{i}^{\alpha}\left(M_{\bar{K}}\right) \mu_{j}^{\beta}\left(N_{\bar{K}}\right)=\sum_{k} \sum_{\gamma} m_{k}^{\gamma} \mu_{k}^{\gamma}\left((M \otimes N)_{\bar{K}}\right)
$$

where, for each $k, \gamma$ runs through the embeddings of $Z_{k}(M \otimes N)$ into $\bar{K}$. This gives a formula as wanted.

It remains to prove that, given a simple factor $A_{k}$ of $A_{i j}$, one may find a formula with $m_{k}>0$. For this, it suffices to show that there is a pair $(\alpha, \beta)$ such that

$$
\operatorname{Hom}_{\bar{K}}\left(\left(e_{i}^{\alpha} \otimes f_{j}^{\beta}\right)\left(A_{i j} \otimes_{K} \bar{K}\right)\left(e_{i}^{\alpha} \otimes f_{j}^{\beta}\right), A_{k} \otimes_{K} \bar{K}\right) \neq 0
$$

This is obvious, since $\operatorname{Hom}_{K}\left(A_{i j}, A_{k}\right) \neq 0$ and $A_{i j} \otimes_{K} \bar{K}=\prod_{\alpha, \beta}\left(e_{i}^{\alpha} \otimes f_{j}^{\beta}\right)\left(A_{i j} \otimes_{K}\right.$ $\left.\bar{K})\left(e_{i}^{\alpha} \otimes f_{j}^{\beta}\right)\right)$.

2.5. Remark. A. del Padrone gives in [16, (5)] an example of two objects $M, N$ of some $\mathcal{A}$ which are geometrically of integral type but such that $M \otimes N$ is not geometrically of integral type. As in Remark 2.3, it is based on Deligne's categories of "representations of the symmetric group with $t$ letters", $t$ a complex number [14]. 
3.1. Definition. Let $\mathcal{A}$ be a rigid $K$-category, $M \in \mathcal{A}$ and $f \in \operatorname{End}(M)$. The zeta function of $f$ is

$$
Z(f, t)=\exp \left(\sum_{n \geq 1} \operatorname{tr}\left(f^{n}\right) \frac{t^{n}}{n}\right) \in K[[t]] .
$$

3.2. Theorem. Suppose that $\mathcal{A}$ is semi-simple and that $M \in \mathcal{A}$ is of integral type. Then,

a) For any $f \in \operatorname{End}(M), Z(f, t) \in K(t)$. More precisely, one has with the notation of Definition 1.1

$$
Z(f, t)=\prod_{i} \operatorname{Nrd}_{A_{i}}\left(e_{i}-e_{i} f t\right)^{-\mu_{i}(M)}
$$

where, for all $i, \operatorname{Nrd}_{A_{i}}\left(e_{i}-e_{i} f t\right):=N_{Z_{i}(M) / K} \operatorname{Nrd}_{A_{i} / Z_{i}(M)}\left(e_{i}-e_{i} f t\right)$ denotes the inverse reduced characteristic polynomial of the element $e_{i} f$ in $A_{i}$.

b) If $f$ is invertible, one has the functional equation

$$
Z\left(f^{-1}, t^{-1}\right)=(-t)^{\chi(M)} \operatorname{det}(f) Z(f, t)
$$

where $\chi(M)=\operatorname{tr}\left(1_{M}\right)$ and $\operatorname{det}(f)=\prod_{i} \operatorname{Nrd}_{A_{i}}\left(e_{i} f\right)^{\mu_{i}(M)}$.

Proof. (For another proof, see Proposition 15.5.) a) Applying the formula of Proposition 1.2, we get

$$
\begin{aligned}
& Z(f, t)=\exp \left(\sum_{n \geq 1} \operatorname{Trd}_{M}\left(\mu(M) f^{n}\right) \frac{t^{n}}{n}\right) \\
&=\exp \left(\sum_{n \geq 1} \sum_{i} \operatorname{Trd}_{M}\left(\mu_{i}(M) e_{i} f^{n}\right) \frac{t^{n}}{n}\right) \\
&=\prod_{i} \exp \left(\sum_{n \geq 1} \operatorname{Trd}_{A_{i}}\left(\left(e_{i} f\right)^{n}\right) \frac{t^{n}}{n}\right)^{\mu_{i}(M)}
\end{aligned}
$$

and the conclusion follows from the well-known linear algebra identity

$$
\exp \left(\sum_{n \geq 1} \operatorname{Trd}_{A_{i}}\left(\left(e_{i} f\right)^{n}\right) \frac{t^{n}}{n}\right)=\operatorname{Nrd}_{A_{i}}\left(e_{i}-e_{i} f t\right)^{-1} \text {. }
$$


For b), we write

$$
\operatorname{Nrd}_{A_{i}}\left(e_{i}-e_{i} f^{-1} t^{-1}\right)=\operatorname{Nrd}_{A_{i}}\left(-e_{i} f^{-1} t^{-1}\right) \operatorname{Nrd}_{A_{1}}\left(e_{i}-e_{i} f t\right)
$$

hence

$$
\begin{aligned}
Z\left(f^{-1}, t^{-1}\right) & =\prod_{i} \operatorname{Nrd}_{A_{i}}\left(e_{i}-e_{i} f^{-1} t^{-1}\right)^{-\mu_{i}(M)} \\
= & \prod_{i} \operatorname{Nrd}_{A_{i}}\left(-e_{i} f^{-1} t^{-1}\right)^{-\mu_{i}(M)} \operatorname{Nrd}_{A_{1}}\left(e_{i}-e_{i} f t\right)^{-\mu_{i}(M)} \\
& =\prod_{i} \operatorname{Nrd}_{A_{i}}\left(-e_{i} f^{-1} t^{-1}\right)^{-\mu_{i}(M)} Z(f, t)
\end{aligned}
$$

and

$$
\begin{aligned}
& \prod_{i} \operatorname{Nrd}_{A_{i}}\left(-e_{i} f^{-1} t^{-1}\right)^{-\mu_{i}(M)}= \\
& \quad(-t)^{\sum_{i} \mu_{i}(M) d_{i}(M) \delta_{i}(M)} \prod_{i} \operatorname{Nrd}_{A_{i}}\left(e_{i} f\right)^{\mu_{i}(M)}=(-t)^{\chi(M)} \operatorname{det}(f) .
\end{aligned}
$$

3.3. Remark. The definition of det shows that

$$
\operatorname{det}(1-f t)=Z(f, t)^{-1}
$$

if the left hand side is computed in $\mathcal{A}_{K(t)}$.

\section{OBJeCts of CONSTANT Sign AND Finite Dimensionality}

4.1. Definition. Let $\mathcal{A}$ be semi-simple of integral type, and let $M \in \mathcal{A}$. We say that $M$ is of constant sign if, in the decomposition of Proposition 1.2, all $\mu_{i}(M)$ have the same sign. This sign is, by definition, the sign of $M$.

4.2. Proposition. Assume that $M$ and $N$ are simple and that, in Theorem 2.4, $M \otimes N$ is of constant sign. Then we have $\left|\mu_{k}(M \otimes N)\right| \leq|\mu(M) \mu(N)|$ for all $k$, and the sign of $M \otimes N$ is the product of the signs of $M$ and $N$. If $|\mu(M)|=$ $|\mu(N)|=1$, then $M \otimes N$ is isotypical. Moreover, $\mu(M \otimes N)=\mu(M) \mu(N)$.

Proof. This follows from the last statement of Theorem 2.4. In the case where $|\mu(M)|=|\mu(N)|=1$, Formula (2.2) gives the conclusion. 
As a variant of the previous proposition, we are going to give a criterion for $\mathcal{A}$ to be a Kimura-O'Sullivan category, i.e. for every object of $\mathcal{A}$ to be finitedimensional. The following lemma has nothing to do with multiplicities, but is quite useful:

4.3. Lemma. Let $\mathcal{A}$ be a pseudo-abelian rigid $K$-category. Let $\bar{K}$ be an algebraic closure of K. If $\mathcal{A}$ is Kimura-O'Sullivan, so is $\mathcal{A}_{\bar{K}}^{\natural}$; the converse is true if $\sqrt[\otimes]{0}=0$ (see [4, déf. 7.4.1]), for example if $\mathcal{A}$ is semi-simple.

Proof. This is not proven in [4] but is easy to check: if $\mathcal{A}$ is Kimura-O'Sullivan, so is $\mathcal{A}_{\bar{K}}^{\natural}$ since any direct summand of a finite-dimensional object is finite-dimensional [4, prop. 9.1.12]. Conversely, suppose $\mathcal{A}_{\bar{K}}^{\natural}$ Kimura-O'Sullivan and let $M \in \mathcal{A}$. Write $M_{\bar{K}}=M^{+} \oplus M^{-}$, with $M^{+}$positive and $M^{-}$negative. Since $\sqrt[\otimes]{0}=0$ in $\mathcal{A}$, the same is true in $\mathcal{A}_{\bar{K}}[4,7.4 .4]$ and the decomposition of $M_{\bar{K}}$ is unique by $[4$, th. 9.2 .1 a) $]$ (or its proof). Therefore, the idempotent of $\operatorname{End}\left(M_{\bar{K}}\right)$ which defines it is Galois-invariant, and descends to an idempotent of $\operatorname{End}(M)$.

4.4. Lemma. Let $\mathcal{A}$ be a semi-simple, pseudo-abelian rigid $K$-category and let $M \in \mathcal{A}$ be finite-dimensional. Then $M$ is positive (resp. negative) if and only if it is of constant positive (resp. negative) sign.

Proof. It is sufficient to prove that, if $M$ is positive (resp. negative), it is of constant positive (resp. negative) sign. For this, we may assume that $K$ is algebraically closed. Every direct summand $N$ of $M$ is positive (resp. negative), and $\chi(N)$ has the sign of $N$ [4, Prop. 7.2.7 ii)]. Hence the result follows from Lemma $1.3 \mathrm{~b})$.

4.5. Proposition. a) Let $\mathcal{A}$ be a semi-simple rigid $K$-category, pseudo-abelian and geometrically of integral type. Then the following conditions are equivalent:

(i) $\mathcal{A}$ is Kimura-O'Sullivan.

(ii) If $M$ and $M^{\prime}$ are of constant sign, $M \otimes M^{\prime}$ is of constant sign.

b) If $K$ is algebraically closed and all multiplicities are equal to \pm 1 , these conditions are also equivalent to

(iii) If $S$ and $S^{\prime}$ are simple, then $S \otimes S^{\prime}$ is simple.

(iv) Every simple object is invertible. 
(Remark 2.3 gives an instance where Conditions (i) and (ii) of a) do not hold.)

Proof. a) (i) $\Rightarrow$ (ii) follows from Lemma 4.4. For the converse, we may assume $K$ algebraically closed, thanks to Lemma 4.3. It is sufficient to prove that a simple object $S$ is either positive or negative. We have $\mu(S)=\chi(S)$ (Lemma $1.3 \mathrm{~b})$ ); recall that this integer is $\neq 0$ by Proposition 1.2.

Suppose that $\chi(S)>0$. By $[4,7.2 .4], \chi\left(\Lambda^{\chi(S)+1}(S)\right)=0$. Writing $\Lambda^{\chi(S)+1}(S)=$ $\bigoplus_{\alpha} S_{\alpha}^{n_{\alpha}}$ with the $S_{\alpha}$ simple and all distinct, we get

$$
0=\sum_{\alpha} n_{\alpha} \chi\left(S_{\alpha}\right)
$$

By hypothesis, $\Lambda^{\chi(S)+1}(S)$ is of constant sign, hence all $n_{\alpha}$ are 0 and $\Lambda^{\chi(S)+1}(S)=$ 0 . The case where $\chi(S)<0$ is similar.

b) (i) $\Rightarrow$ (iv): by hypothesis, simple objects are 1-dimensional (Definition 11.1), hence invertible by Lemma 11.2 a) below.

(iv) $\Rightarrow$ (iii): this is obvious.

(iii) $\Rightarrow$ (ii): this follows from Proposition 4.2, which says that $\mu\left(S \otimes S^{\prime}\right)=$ $\mu(S) \mu\left(S^{\prime}\right)$.

\section{Multiplicities in Rigid tensor CATEgories of HOMOlogical type}

5.1. Definition. a) A rigid $K$-category $\mathcal{A}$ is of homological type if there exists a tensor functor

$$
H: \mathcal{A} \rightarrow \operatorname{Vec}_{L}^{ \pm}
$$

where $L$ is an extension of $K$ and $\operatorname{Vec}_{L}^{ \pm}$is the tensor category of $\mathbf{Z} / 2$-graded finitedimensional $L$-vector spaces, provided with the Koszul rule for the commutativity constraint. We say that $H$ is a realisation of $\mathcal{A} .^{2}$

We say that $\mathcal{A}$ is neutrally of homological type if one may choose $L=K$.

b) A semi-simple rigid $K$-category $\overline{\mathcal{A}}$ is of homological origin (resp. neutrally of homological origin) if it is $\otimes$-equivalent to $\mathcal{A} / \mathcal{N}$, where $\mathcal{A}$ is a rigid $K$-category of homological type (resp. neutrally of homological type) and $\mathcal{N}=\mathcal{N}(\mathcal{A})$ is the ideal of morphisms universally of trace 0 (see (1.2)).

\footnotetext{
${ }^{2}$ When $\mathcal{A}$ is abelian, this is what Deligne calls a super-fibre functor in [13], except that we do not require any exactness or faithfulness property here.
} 
As this will be used throughout, we recall that the functor $H$ is compatible with (categorical) traces: for any $M \in \mathcal{A}$ and any $f \in \operatorname{End}_{\mathcal{A}}(M), \operatorname{tr}(f)=\operatorname{tr} H(f)$ via the injection $K \hookrightarrow L$. This is the "Lefschetz trace formula" in this abstract context.

5.2. Lemma. If $\mathcal{A}$ is of homological type, $\mathcal{A} / \mathcal{N}$ is semi-simple. If moreover it is neutrally of homological type and the corresponding realisation $H$ is faithful, the functor $\mathcal{A} \rightarrow \mathcal{A} / \mathcal{N}$ has the idempotent lifting property.

Proof. The first statement follows from [5, Th. 1 a)]. For the second, let $M \in \mathcal{A}$ and $\bar{M}$ be its image in $\overline{\mathcal{A}}$. The hypothesis implies that $\operatorname{End}_{\mathcal{A}}(M)$ is a finitedimensional $K$-algebra. Let $\mathcal{R}$ be its radical: it is nilpotent and contained in $\mathcal{N}(M, M)$ by $\left[5\right.$, Th. 1 a)]. Thus $\operatorname{End}_{\overline{\mathcal{A}}}(\bar{M})$ is a quotient of the semi-simple algebra $\operatorname{End}_{\mathcal{A}}(M) / \mathcal{R}$. Therefore we may lift orthogonal idempotents of $\operatorname{End}_{\overline{\mathcal{A}}}(\bar{M})$ to orthogonal idempotents of $\operatorname{End}_{\mathcal{A}}(M)$, first in $\operatorname{End}_{\mathcal{A}}(M) / \mathcal{R}$ and then in $\operatorname{End}_{\mathcal{A}}(M)$ itself.

5.3. Notation. Let $\mathcal{A}$ be of homological type. For $M \in \mathcal{A}$, we write $\delta_{i}(M), d_{i}(M)$, $\mu_{i}(M)$ for $\delta_{i}(\bar{M}), d_{i}(\bar{M}), \mu_{i}(\bar{M})$, where $\bar{M}$ is the image of $M$ in $\overline{\mathcal{A}}$.

5.4. Lemma. Let $E$ be an extension of $K$. If $\overline{\mathcal{A}}$ is of homological origin, then $\overline{\mathcal{A}}_{E}:=\overline{\mathcal{A}} \otimes_{K} E$ is also of homological origin.

Proof. Let $\mathcal{A}$ of homological type be such that $\mathcal{A} / \mathcal{N} \simeq \overline{\mathcal{A}}$, and let $H: \mathcal{A} \rightarrow \operatorname{Vec}_{L}^{ \pm}$ be a realisation of $\mathcal{A}$. Consider the tensor functor

$$
H_{E}: \mathcal{A}_{E} \rightarrow \operatorname{Vec}_{L \otimes_{K} E}^{ \pm}
$$

given by $H_{E}(M)=H(M) \otimes_{K} E$. Here $L \otimes_{K} E$ is not a field in general, but we can map it to one of its residue fields $L^{\prime}$. Then the composite functor

$$
H^{\prime}: \mathcal{A}_{E} \rightarrow \operatorname{Vec}_{L^{\prime}}^{ \pm}
$$

is a tensor functor. To conclude, it suffices to observe that $\overline{\mathcal{A}}_{E} \simeq \mathcal{A}_{E} / \mathcal{N}\left(\mathcal{A}_{E}\right)$ by $[5$, Lemme 1].

5.5. Lemma. Suppose that $\overline{\mathcal{A}}$ is neutrally of homological origin. Then the pseudoabelian envelope of $\overline{\mathcal{A}}$ is also neutrally of homological origin.

Proof. If $\overline{\mathcal{A}}$ is neutrally of homological origin, one may find a faithful realisation $H: \mathcal{A} \rightarrow \operatorname{Vec}_{K}^{ \pm}$. This realisation extends to the pseudo-abelian envelope $\mathcal{A}^{\natural}$ of 
$\mathcal{A}$, since $\operatorname{Vec}_{K}^{ \pm}$is pseudo-abelian. On the other hand, Lemma 5.2 implies that $\mathcal{A}^{\natural} / \mathcal{N}^{\natural}$ is pseudo-abelian, where $\mathcal{N}^{\natural}$ is the ideal $\mathcal{N}$ of $\mathcal{A}^{\natural}$; but the obvious functor $\mathcal{A} / \mathcal{N} \rightarrow \mathcal{A}^{\natural} / \mathcal{N}^{\natural}$ is clearly a pseudo-abelian envelope.

5.6. Theorem. If $\overline{\mathcal{A}}$ is of homological origin, any $\bar{M} \in \overline{\mathcal{A}}$ is Schur-finite; in particular, $\overline{\mathcal{A}}$ is geometrically of integral type.

Proof. Choose $\left(\mathcal{A}, H: \mathcal{A} \rightarrow \operatorname{Vec}_{L}^{ \pm}\right)$as in the proof of Lemma 5.4. As before, we may assume that $H$ is faithful. Lift $\bar{M}$ to $M \in \mathcal{A}$. Then $H(M)$ is finitedimensional, hence Schur-finite, which implies that $M$ and therefore $\bar{M}$ is Schurfinite. The conclusion now follows from Proposition $2.2 \mathrm{e}$ ).

5.7. Remark. The converse of Theorem 5.6 holds. Namely, if every object of $\overline{\mathcal{A}}$ is Schur-finite, then the same is true in $\left(\overline{\mathcal{A}}_{\bar{K}}\right)^{\natural}$. By [13, 0.6 and following remark], $\left(\overline{\mathcal{A}}_{\bar{K}}\right)^{\natural}$ is $\otimes$-equivalent to $\operatorname{Rep}(G, \varepsilon)$, where $(G, \varepsilon)$ is a super-affine group scheme over $\bar{K}$; in particular, $\left(\overline{\mathcal{A}}_{\bar{K}}\right)^{\natural}$ and hence $\overline{\mathcal{A}}$ admits a realisation into $\operatorname{Vec}_{\bar{K}}^{ \pm}$. This shows that if $\overline{\mathcal{A}}$ is of homological origin, then it is actually of homological type. Another approach to this idea is the one in [3], using $\otimes$-sections.

However, in the case of pure motives, one wants of course to study the general situation of Definition $5.1 \mathrm{~b}$ ), using "natural" realisation functors! This is what we do in the remainder of this section.

5.8. Definition. Let $\mathcal{A}$ be of homological type, and let $H: \mathcal{A} \rightarrow \operatorname{Vec}_{L}^{ \pm}$be a realisation functor. Given $M \in \mathcal{A}$, we say that $M$ has the sign property (with respect to $H$ ) if there exists $p \in \operatorname{End}_{\mathcal{A}}(M)$ such that $H(p)$ is the identity on $H^{+}(M)$ and is 0 on $H^{-}(M)$.

5.9. Lemma (cf. [4, 9.2.1]). With the notation of Definition 5.8:

a) If $M$ is finite-dimensional, it has the sign property.

b) The converse is true if $H$ is faithful and $\mathcal{N}(M, M)$ is a nilideal (the latter is automatic if $L=K$ ).

5.10. Proposition. Let $\mathcal{A}$ be of homological type, $\overline{\mathcal{A}}:=\mathcal{A} / \mathcal{N}$ and let $H: \mathcal{A} \rightarrow$ $\operatorname{Vec}_{L}^{ \pm}$be a realisation functor. Then

a) For any simple object $S \in\left(\overline{\mathcal{A}}_{L}\right)^{\natural}, d(S) \mid \mu(S)$.

b) Suppose $H$ faithful. Let $M \in \mathcal{A}$ have the sign property. Then the nilpotence level $r$ of $\mathcal{N}(M, M)$ verifies

$$
r<\prod_{i}\left(\frac{\left|\mu_{i}(M)\right|}{d_{i}(M)}+1\right)
$$


where $d_{i}(M)=d\left(S_{i}\right)$, with $S_{i}$ a simple summand of $\bar{M}_{L} \in \overline{\mathcal{A}}_{L}^{\natural}$ corresponding to its $i$-th isotypical component (see Remark 1.4).

c) If $\bar{M}$ is isotypical and $\mu(M)= \pm 1$, then $\mathcal{N}(M, M)=0$.

Proof. a) Since $\mathcal{A}_{L} / \mathcal{N}\left(\mathcal{A}_{L}\right)=\overline{\mathcal{A}}_{L}, \overline{\mathcal{A}}_{L}$ is neutrally of homological origin; up to quotienting $\mathcal{A}_{L}$ and replacing $K$ by $L$, we may assume $L=K$ and $H$ faithful. Then $\mathcal{N}(M, M)$ is nilpotent for all $M$ and, as in the proof of Lemma 5.5, we may further assume that $\mathcal{A}$ and $\overline{\mathcal{A}}$ are pseudo-abelian.

Let $\tilde{S} \in \mathcal{A}$ be mapping to $S$. By Lemma 5.2 and Wedderburn's section theorem, the map $\operatorname{End}_{\mathcal{A}}(\tilde{S}) \rightarrow \operatorname{End}_{\overline{\mathcal{A}}}(S)$ has a ring-theoretic section $\sigma$. This makes $H(\tilde{S})$ a module over the division ring $\operatorname{End}_{\overline{\mathcal{A}}}(S)$. Therefore $\operatorname{dim}_{K} H^{\varepsilon}(\tilde{S})$ is divisible by $\operatorname{dim}_{K} \operatorname{End}_{\overline{\mathcal{A}}}(S)=\delta(S) d(S)^{2}$ for $\varepsilon= \pm 1$. On the other hand,

$$
\operatorname{dim}_{K} H^{+}(\tilde{S})-\operatorname{dim}_{K} H^{-}(\tilde{S})=\mu(S) \delta(S) d(S)
$$

by Proposition 2.2 a). Therefore, $\delta(S) d(S)^{2}$ divides $\mu(S) \delta(S) d(S)$, which means that $d(S)$ divides $\mu(S)$, as claimed.

b) Assume first $L=K$. Without loss of generality, we may also assume $\mathcal{A}$ pseudoabelian. Let $\mathcal{N}_{M}=\mathcal{N}(M, M)$ and consider the filtration $\left(\left(\mathcal{N}_{M}\right)^{i} H(M)\right)_{0 \leq i \leq r-1}$. Note that $\left(\mathcal{N}_{M}\right)^{i} H(M)=\left(\mathcal{N}_{M}\right)^{i+1} H(M) \Longleftrightarrow\left(\mathcal{N}_{M}\right)^{i}=0$ since $\mathcal{N}_{M}$ is a nilpotent set of endomorphisms of $H(M)$. The associated graded $\left(\operatorname{gr}^{i} H(M)\right)_{0 \leq i \leq r-1}$ is a graded $\operatorname{End}_{\overline{\mathcal{A}}}(\bar{M})$-module, and $\operatorname{gr}^{i} H(M) \neq 0$ for all $i<r$.

Since $M$ has the sign property, for each isotypical component $\bar{M}_{i}$ of $\bar{M}$, with lift $M_{i}$ in $\mathcal{A}$ (Lemma 5.2), one has either $H^{+}\left(M_{i}\right)=0$ or $H^{-}\left(M_{i}\right)=0$. The proof of a) then shows that $\operatorname{gr} H\left(M_{i}\right)$ is an $\operatorname{End}_{\overline{\mathcal{A}}}\left(\bar{M}_{i}\right)$-module of length $\frac{\left|\mu\left(S_{i}\right)\right|}{d\left(S_{i}\right)}$ where $S_{i}$ is the associated simple object. Note that $\operatorname{End}_{\overline{\mathcal{A}}}(\bar{M})=\prod_{i} \operatorname{End}_{\overline{\mathcal{A}}}\left(\bar{M}_{i}\right)$ : it follows that $\operatorname{gr} H(M)$ is an $\operatorname{End}_{\overline{\mathcal{A}}}(\bar{M})$-module of length $\prod\left(\frac{\left|\mu\left(S_{i}\right)\right|}{d\left(S_{i}\right)}+1\right)-1 .^{3}$ Hence the inequality.

In general we extend scalars from $K$ to $L$. Let $s=\prod_{i}\left(\frac{\left|\mu_{i}(M)\right|}{d_{i}(M)}+1\right)-1$. Applying the result to the category $\mathcal{A} L$ with the same objects as $\mathcal{A}$ and such that $\mathcal{A} L(M, N)=H(\mathcal{A}(M, N)) L \subseteq \operatorname{Hom}_{L}(H(M), H(N))$, we get $(\mathcal{N}(M, M) L)^{s}=0$, hence $\mathcal{N}(M, M)^{s} \subseteq(\mathcal{N}(M, M) L)^{s}=0$.

c) This follows immediately from b).

${ }^{3} \overline{\text { If } A_{i} \text { are rings and }} M_{i}$ are $A_{i}$-modules of finite lengths $l_{i}$, the $\prod A_{i}$-length of $\prod M_{i}$ is $\prod_{i}\left(l_{i}+1\right)-1$. 
5.11. Remark. In case $M$ is finite dimensional, we have another bound for the nilpotence level of $\mathcal{N}(M, M)$ (valid without assuming $H$ faithful). For simplicity, suppose that $M$ is either positive or negative, and let $n=|\chi(M)|$. By [40, Corollary 10.2], $f^{n}=0$ for all $f \in \mathcal{N}(M, M)$, which implies that $\mathcal{N}(M, M)^{n^{2}}=0$ by a theorem of Razmyslov improving the Nagata-Higman bound [51, 4.3]. I learned of this improvement from Alessio del Padrone, who has generalised this bound to any finite-dimensional $M$ with $n=\left|\chi\left(M^{+}\right)\right|+\left|\chi\left(M^{-}\right)\right|[15,2.4 .10]$.

These two bounds have completely different behaviours: in Proposition $5.10 \mathrm{c}$ ) the former is optimal while the latter is not, but on the other hand if $M$ is a direct sum of $n$ invertible objects pairwise non-isomorphic, the bound of Proposition $5.10 \mathrm{~b}$ ) is $2^{n}-1$ while the other one is $n^{2}$.

As del Padrone pointed out, a third unrelated nilpotency bound is the one predicted by the Bloch-Beilinson-Murre conjecture for the Chow motive of a smooth projective variety $X$ (namely, $\operatorname{dim}(X)+1$ [30, Strong conj. 2.1]).

5.12. Remark. Coming back to the zeta function of an endomorphism, suppose that $\mathcal{A}$ is of homological type; let $M \in \mathcal{A}$ and $f \in \operatorname{End}_{\mathcal{A}}(M)$. If $H$ is a realisation of $\mathcal{A}$, we have by the usual computation

$$
Z(f, t)=\operatorname{det}(1-H(f) t)^{-1}=\frac{\operatorname{det}\left(1-f t \mid H^{-}(M)\right)}{\operatorname{det}\left(1-f t \mid H^{+}(M)\right)} .
$$

Let $\bar{M}$ be the image of $M$ in $\overline{\mathcal{A}}=\mathcal{A} / \mathcal{N}$ and $\bar{f}$ be the image of $f$ in $\operatorname{End}_{\overline{\mathcal{A}}}(\bar{M})$. Since $Z(f, t)=Z(\bar{f}, t)$, we get from Theorem 5.6 and Theorem 3.2 a) the identity

$$
\frac{\operatorname{det}\left(1-f t \mid H^{+}(M)\right)}{\operatorname{det}\left(1-f t \mid H^{-}(M)\right)}=\prod_{i} \operatorname{Nrd}_{A_{i}}\left(e_{i}-e_{i} \bar{f} t\right)^{\mu_{i}(M)} \text {. }
$$

Suppose for example that $\bar{M}$ is simple; the identity reduces to

$$
\frac{\operatorname{det}\left(1-f t \mid H^{+}(M)\right)}{\operatorname{det}\left(1-f t \mid H^{-}(M)\right)}=\operatorname{Nrd}_{A}(1-\bar{f} t)^{\mu(M)}
$$

where $A=\operatorname{End}_{\overline{\mathcal{A}}}(\bar{M})$.

Supposing further that $\mu(M)>0$ to fix ideas, we find that the inverse characteristic polynomial of $f$ acting on $H^{-}(M)$ (with coefficients in $L$ ) divides the one for $H^{+}(M)$, and the quotient has coefficients in $K$. This does not imply, however, that $H^{-}(M)=0$. 


\section{EXAMPLES}

In our first example, let $\mathcal{A}$ be the rigid $K$-category of vector bundles over $\mathbb{P}_{K}^{1}$. It is of homological type, with realisation functor $H: \mathcal{A} \rightarrow \operatorname{Vec}_{L}$ for $L=K(t)$ given by the generic fibre. Its indecomposable objects are the $\mathcal{O}(n)$ for $n \in \mathbf{Z}$ : they are all of multiplicity 1 but $\mathcal{A}(\mathcal{O}(p), \mathcal{O}(q))=\mathcal{N}(\mathcal{O}(p), \mathcal{O}(q)) \neq 0$ whenever $p<q$. This shows that the condition "isotypical" is necessary in Proposition $5.10 \mathrm{c}$ ) (I am indebted to Yves André for pointing out this example). If one extends scalars from $K$ to $L$ in the style of the proof of this proposition, one finds $\mathcal{A} L(\mathcal{O}(p), \mathcal{O}(q))=L$ whenever $p<q$.

Our main source of examples is, of course, the category $\mathcal{M}_{\text {num }}(k)$ of pure motives over a field $k$ modulo numerical equivalence. By Jannsen's theorem [29] and Theorem 5.6, $\mathcal{M}_{\text {num }}(k)$ is semi-simple and geometrically of integral type. We shall compute the multiplicities in certain cases.

6.1. Remark. Let $X$ be smooth projective and suppose that $h(X)$ admits a Künneth decomposition $h(X) \simeq \bigoplus_{i=0}^{2 d} h^{i}(X)$, relatively to a Weil cohomology $H$. Using $H$, one sees that $h^{i}(X)$ is finite-dimensional, and positive or negative according to the parity of $i$. By Lemma 4.4, this shows that $h^{i}(X)$ is of constant $\operatorname{sign}(-1)^{i}$.

We leave it to the reader to check that the multiplicities of Artin (even ArtinTate) motives are always +1 . The next case is that of abelian varieties.

Let $A$ be an abelian variety of dimension $g$ over $k$. Then we have the Künneth decomposition

$$
h(A) \simeq \bigoplus_{i=0}^{2 g} h^{i}(A)
$$

with $h^{i}(A) \simeq S^{i}\left(h^{1}(A)\right)[55]$. Moreover,

$$
\text { End } h^{1}(A)=\operatorname{End}^{0}(A):=\operatorname{End}(A) \otimes \mathbf{Q} .
$$

Finally, $\chi\left(h^{1}(A)\right)=-2 g$. From this and Proposition 2.2, we get for $A$ simple:

$$
\mu\left(h^{1}(A)\right)=-\frac{2 g}{\delta\left(\operatorname{End}^{0}(A)\right) d\left(\operatorname{End}^{0}(A)\right)} .
$$

We recover the fact that the denominator divides the numerator. 
Like Milne [47], we shall say that $A$ has many endomorphisms if

$$
\sum_{i} \delta\left(\operatorname{End}^{0}\left(A_{i}\right)\right) d\left(\operatorname{End}^{0}\left(A_{i}\right)\right)=2 g
$$

where $A_{i}$ runs through the simple factors of $A$, or equivalently if

$$
\delta\left(\operatorname{End}^{0}\left(A_{i}\right)\right) d\left(\operatorname{End}^{0}\left(A_{i}\right)\right)=2 g_{i}
$$

for all $i$, where $g_{i}=\operatorname{dim} A_{i}$. This terminology is less ambiguous than "having complex multiplication".

6.2. Definition. Let $M \in \mathcal{M}_{\text {num }}(k)$ be a pure motive modulo numerical equivalence. Then $M$ is of abelian type if it is isomorphic to a direct summand of the tensor product of an Artin motive and the motive of an abelian variety.

Motives of abelian type are stable under direct sums, direct summands, tensor products and duals. We then have:

6.3. Theorem. a) For $A$ a simple abelian variety, $\mu\left(h^{1}(A)\right)=-1$ if and only if $A$ has many endomorphisms.

b) If $g=1$, then $\mu\left(h^{1}(A)\right)= \begin{cases}-1 & \text { if } A \text { has complex multiplication } \\ -2 & \text { otherwise. }\end{cases}$

c) If $A$ has many endomorphisms, all multiplicities of $h^{i}(A)$ are equal to $(-1)^{i}$.

d) If $k$ is a finite field, then the multiplicities of any motive of abelian type are equal to \pm 1 .

Proof. a) and b) are clear; c) follows from a) and Proposition 4.2 (see Remark 6.1 ), and d) follows from c) since any abelian variety over a finite field has many endomorphisms [56].

The next interesting case is that of $t_{2}(S)$ where $S$ is a surface [35]. If $k=\mathbf{C}$, there are many examples where the Hodge realisation of $t_{2}(S)$ is absolutely simple [50, Ex. 5 and Cor. 18]. A fortiori $t_{2}(S)$ is absolutely simple, and Proposition 2.2 a) shows that its multiplicity equals its Euler characteristic, i.e. $b^{2}-\rho$ where $b^{2}$ is the second Betti number and $\rho$ is the Picard number. 


\section{Part II. Number of points of function fields over finite fields}

\section{INTRODUCTION}

Let $k$ be a field; if $\sim$ is an adequate equivalence relation on algebraic cycles, we denote by $\mathcal{M}_{\sim}(k)$ or simply $\mathcal{M}_{\sim}$ the category of motives modulo $\sim$ with rational coefficients, and by $\mathcal{M}_{\sim}^{\text {eff }}$ its full subcategory consisting of effective motives [53]. ${ }^{4}$ We use the convention that the functor $X \mapsto h(X)$ from smooth projective $k$ varieties to $\mathcal{M}_{\sim}^{\text {eff }}$ is covariant. Examples: rational equivalence (rat), numerical equivalence (num) or homological equivalence with respect to a Weil cohomology theory $H$.

Using the point of view of birational motives (developed jointly with Sujatha [36]), we give a simple proof of a result of Esnault on the existence of rational points for smooth projective varieties with "trivial" Chow group of zero-cycles over a finite field $\mathbf{F}_{q}$ [19]. More generally, we prove in Corollary 9.6 that if two smooth projective $\mathbf{F}_{q}$-varieties $X, Y$ mutually dominate each other up to varieties with trivial $\mathrm{CH}_{0}$, they have the same number of rational points modulo $q$. We also recover the "constant" theorem of Fahkruddin and Rajan [22, Cor. 1.3] in the projective case, but not their main theorem "in families" (ibid., Th. 1.1) or Esnault's most general theorem in this direction [20]. (It seems that a motivic approach to these two results along the same lines should involve something like one of Ayoub's specialisation functors on Voevodsky's triangulated categories of motives [7].) We also show that if $X$ is dominated by the product of a supersingular abelian variety and a smooth projective variety with trivial $C H_{0}$, then $X$ has a rational point over $\mathbf{F}_{q}$ (Corollary 9.7). One could imagine other examples.

Some of these results were originally obtained via $p$-adic cohomology: in particular the proofs in [19] use the main properties of rigid cohomology of open varieties (finite-dimensionality, Gysin exact sequences and the theory of slopes). The proofs given here are very simple-minded; they use the existence of a Weil cohomology theory (for smooth projective varieties) and de Jong's alteration theorem.

The idea of considering effective motives and their divisibility by the Lefschetz motive was anticipated by Serre [54]. The story of the birational invariance of

\footnotetext{
${ }_{4}^{4}$ With notation as in [53], an object of $\mathcal{M}_{\sim}$ is effective if it is isomorphic to an triple $(X, p, n)$ with $n \leq 0$; one may then find such a triple with $n=0$.
} 
the number of points modulo $q$ (a special case of Corollary 9.6) is amusing: this question had initially been raised by Kollár and the 3-dimensional case was proven by Lachaud and Perret in [45]. Then I independently found a proof in general as an application of birational motives [31]. However, as was then pointed out by Chambert-Loir, it in fact follows from much earlier work of Ekedahl [18]. See Chambert-Loir's Bourbaki talk [9] for details as well as nonabelian applications of $p$-adic cohomology, not covered by the methods of this paper.

In Section 7 we review results on birational motives from [36] and add some remarks. In Section 8 we show that the number of points of an effective motive, as defined in [42], is an integer (it is a priori a rational number): see Theorem 8.1. In Section 9 we prove the main result (Theorem 9.1) which says that the number of points modulo $q$ of a numerical birational motive over $\mathbf{F}_{q}$ is well-defined, and draw the corollaries indicated above. Finally, in Section 10, we show that the Tate conjecture is equivalent to the statement that a numerical birational motive is determined by its number of points over $\mathbf{F}_{q^{n}}$ modulo $q^{n}$ for all $n$ (Corollary 10.2), and point out that the Tate conjecture implies the generalised Tate conjecture over finite fields (Remark 10.32 )).

\section{Birational Motives}

7.1. Definition. The category $\mathcal{M}_{\sim}^{\mathrm{o}}$ is the Karoubian envelope (or idempotent completion) of the quotient of $\mathcal{M}_{\sim}^{\text {eff }}$ by the ideal $J$ consisting of morphisms factoring through an object of the form $M \otimes \mathbb{L}$, where $\mathbb{L}$ is the Lefschetz motive. This is a tensor additive category. If $M \in \mathcal{M}_{\sim}^{\text {eff }}$, we denote by $\bar{M}$ its image in $\mathcal{M}_{\sim}^{\mathrm{o}}$.

7.2. Lemma ([36, Lemmas 5.3 and 5.4]). Let $X, Y$ be two smooth projective irreducible $k$-varieties. Then, in $\mathcal{M}_{\text {rat }}^{\mathrm{o}}$, we have

$$
\operatorname{Hom}(\bar{h}(X), \bar{h}(Y))=C H_{0}\left(Y_{k(X)}\right) \otimes \mathbf{Q} .
$$

Let us briefly recall the proof: for $X, Y$ smooth projective, let $I(X, Y)$ be the subgroup of $C H^{\operatorname{dim} Y}(X \times Y) \otimes \mathbf{Q}$ formed of those correspondences which vanish on $U \times Y$ for some dense open subset $U$ of $X$. Then $I$ is an ideal in the category of rational Chow correspondences: the proof [36, Lemma 5.3] is a slight generalisation of the argument in [23, Ex. 16.1.11]. It is even monoidal, and extends to a monoidal ideal $I$ in $\mathcal{M}_{\text {rat }}^{\text {eff }}$, which obviously contains $J$. Using de 
Jong's theorem [28, Th. 4.1], one sees that $I \otimes \mathbf{Q}=J \otimes \mathbf{Q}$ [36, Lemma 5.4]. In characteristic 0 , one may remove the coefficients $\mathbf{Q}$ by using Hironaka's resolution of singularities. ${ }^{5}$

7.3. Example. Let $X$ be smooth and projective over $k$. Then $\bar{h}(X) \simeq \mathbf{1}$ in $\mathcal{M}_{\text {rat }}^{\mathrm{o}}$ if and only if $C H_{0}\left(X_{k(X)}\right) \otimes \mathbf{Q} \simeq \mathbf{Q}\left(\right.$ write $h(X) \simeq \mathbf{1} \oplus h(X)_{\geq 1}$ in $\left.\mathcal{M}_{\text {rat }}^{\text {eff }}\right)$. In particular, $\bar{h}(X) \simeq \mathbf{1}$ if $X$ is [geometrically] unirational, as expected in [54]. More generally, any separably rationally connected variety has this property. The converse is not true: an Enriques surface $X$ verifies $\bar{h}(X) \simeq \mathbf{1}$ by [8], but is not rational, hence not unirational over a field of characteristic 0 because $\operatorname{Pic}(X)$ contains a $\mathbf{Z} / 2$ summand (recall that any unirational surface is rational by Lüroth's theorem; I thank Colliot-Thélène for pointing out this counterexample).

7.4. Remark. If $K$ is the function field of a smooth projective variety $X$, we may define a motive $\bar{h}(K) \in \mathcal{M}_{\text {rat }}^{\mathrm{o}}$ as follows. If $Y$ is another smooth projective model of $K$, then [the closure of] the graph of a birational isomorphism from $X$ to $Y$ defines an isomorphism $\bar{h}(X) \stackrel{\sim}{\longrightarrow} \bar{h}(Y)$. If there is a third model $Z$, then the system of these isomorphisms is transitive, so defining $\bar{h}(K)$ as the direct limit of the $\bar{h}(X)$ for this type of isomorphisms makes sense and is canonically isomorphic to any of the $\bar{h}(X)$. This construction is functorial for inclusions of fields. If char $k=0$, it is even functorial for $k$-places by [36, Lemma 5.6], although we won't use this. (Extending it to arbitrary function fields in characteristic $p$ would demand more work.)

Note that if $K \subseteq L$, then $\bar{h}(K)$ is a direct summand of $\bar{h}(L)$ : to see this, write $L$ as a finite extension of a purely transcendental extension $E$ of $K$. Then $\bar{h}(E) \stackrel{\sim}{\longrightarrow} \bar{h}(K)$, hence we are reduced to the case where $L / K$ is finite, and then it follows from a transfer argument.

We can slightly generalise this remark as follows:

7.5. Lemma. Let $E / F$ be an extension, where $E$ and $F$ are finitely generated over $k$. Then, for any smooth projective $k$-variety $X$, the map $C H_{0}\left(X_{F}\right) \otimes \mathbf{Q} \rightarrow$ $C H_{0}\left(X_{E}\right) \otimes \mathbf{Q}$ has a retraction which is functorial in $X$.

\footnotetext{
${ }_{5}^{5}$. Chambert-Loir and N. Fakhruddin independently pointed out that Chow's moving lemma is incorrectly applied in the proof of [36, Lemma 5.4]; however, Fakhruddin also pointed out that one avoids Chow's moving lemma by taking the subvariety $Z$ minimal in this proof.
} 
Proof. It suffices to treat the cases where $E / F$ is finite and where $E=F(t)$. In the first case, we may use a trace map. In the second case, $C H_{0}\left(X_{E}\right)$ is a quotient of $C H_{0}\left(X_{F} \times \mathbf{A}_{F}^{1}\right)=C H_{0}\left(X_{F}\right)$, so the map is an isomorphism.

7.6. Proposition. Let $f: X \rightarrow Y$ be a rational map between smooth projective $k$-varieties, and let $f_{*}$ denote the correspondence defined by the graph of $f$. Suppose that $f_{*}: C H_{0}\left(X_{K}\right) \otimes \mathbf{Q} \rightarrow C H_{0}\left(Y_{K}\right) \otimes \mathbf{Q}$ is an isomorphism for some field $K$ containing $k(X)$ and $k(Y)$. Then $f_{*}: \bar{h}(X) \rightarrow \bar{h}(Y)$ is an isomorphism in $\mathcal{M}_{\text {rat }}^{\text {o }}$.

Proof. For a general field $F$ containing $k$, let $N(F)$ and $C(F)$ denote respectively the kernel and the cokernel of $f_{*}: C H_{0}\left(X_{F}\right) \otimes \mathbf{Q} \rightarrow C H_{0}\left(Y_{F}\right) \otimes \mathbf{Q}$. If $E / F$ is a finitely generated extension, Lemma 7.5 implies that $N(F) \rightarrow N(E)$ and $C(F) \rightarrow$ $C(E)$ are split injective. Hence, if we relax the finite generation assumption, these maps are still injective, because Chow groups commute with filtering direct limits of field extensions. It follows that the hypothesis of the proposition is true for $F=k(X)$ and $F=k(Y)$. Interpreting both sides are Hom groups in $\mathcal{M}_{\text {rat }}^{\mathrm{o}}$, we get the conclusion by Yoneda's lemma.

For $\sim=$ num, the category $\mathcal{M}_{\sim}^{o}$ is abelian semi-simple [29]. From [4, Prop. 2.1.7], we therefore get:

7.7. Proposition. a) The projection functor $\pi: \mathcal{M}_{\text {num }}^{\mathrm{eff}} \rightarrow \mathcal{M}_{\text {num }}^{\mathrm{o}}$ is essentially surjective (i.e. taking the karoubian envelope is irrelevant in the definition of $\left.\mathcal{M}_{\text {num }}^{\mathrm{o}}\right)$.

b) $\pi$ has a section $i$ which is also a left and right adjoint.

c) The category $\mathcal{M}_{\text {num }}^{\mathrm{eff}}$ is the coproduct of $\mathcal{M}_{\mathrm{num}}^{\mathrm{eff}} \otimes \mathbb{L}$ and $i\left(\mathcal{M}_{\mathrm{num}}^{\mathrm{o}}\right)$, i.e. any object of $\mathcal{M}_{\mathrm{num}}^{\mathrm{eff}}$ can be uniquely written as a direct sum of objects of these two subcategories.

d) The sequence

$$
0 \rightarrow K_{0}\left(\mathcal{M}_{\text {num }}^{\text {eff }}\right) \stackrel{\cdot \mathbb{L}}{\longrightarrow} K_{0}\left(\mathcal{M}_{\text {num }}^{\text {eff }}\right) \stackrel{\pi_{*}}{\longrightarrow} K_{0}\left(\mathcal{M}_{\text {num }}^{\mathrm{o}}\right) \rightarrow 0
$$

is split exact.

(In d), the injectivity on the left corresponds to the fact that the functor $-\otimes \mathbb{L}$ is fully faithful.) 
7.8. Remarks. 1) In $\mathcal{M}_{\text {num }}^{\mathrm{o}}$, we can extend the end of Remark 7.4 as follows, using semi-simplicity: let $K, L$ be two function fields of smooth projective varieties such that $K \hookrightarrow L\left(t_{1}, \ldots, t_{m}\right)$ and $L \hookrightarrow K\left(t_{1}, \ldots, t_{n}\right)$ for some $m, n$. Then $\bar{h}(K) \simeq$ $\bar{h}(L)$. To get such a result in $\mathcal{M}_{\text {rat }}^{\mathrm{o}}$, one would need to have enough information on the algebra $\operatorname{End}(\bar{h}(K))$.

2) Proposition $7.7 \mathrm{~b}$ ) shows via Remark 7.4 that to a function field $K / k$ one may canonically associate an effective numerical motive $h(K) \in \mathcal{M}_{\text {num }}^{\text {eff }}$, which is a direct summand of $h(X)$ for any smooth projective model $X$ of $K$.

3) One can use [27, Appendix] to show that, contrary to the case of numerical equivalence, the projection functor $\mathcal{M}_{\text {rat }}^{\text {eff }} \rightarrow \mathcal{M}_{\text {rat }}^{\mathrm{o}}$ does not have a right adjoint: compare [35, Remark 14.8.7].

\section{An integrality theorem}

From now on, $k=\mathbf{F}_{q}$ is a field with $q$ elements. Then, for all $n \geq 1$, the assignment

$$
X \mapsto\left|X\left(\mathbf{F}_{q^{n}}\right)\right|=\operatorname{deg}\left(\Delta_{X} \cdot F_{X}^{n}\right)
$$

for a smooth projective variety $X$, where $\Delta_{X}$ is the class of the diagonal and $F_{X}$ is the Frobenius endomorphism viewed as a correspondence, extends to a ring homomorphism

$$
\#_{n}: K_{0}\left(\mathcal{M}_{\text {num }}^{\text {eff }}\right) \rightarrow \mathbf{Q}
$$

by the rule $\#_{n}(X, p)=\operatorname{deg}\left(p \cdot F_{X}^{n}\right)$ if $p=p^{2} \in \operatorname{End}(h(X))$, cf. [42, p. 80].

8.1. Theorem. The homomorphisms $\#_{n}$ take their values in $\mathbf{Z}$.

Proof. It is enough to prove this for $n=1$. More conceptually, we have $\operatorname{deg}(p$. $\left.F_{X}\right)=\operatorname{tr}\left(p \circ F_{X}\right)$ in the rigid tensor category $\mathcal{M}_{\text {num. }}$. We offer two proofs: the original one from [31] and a more elementary one which uses the multiplicities of Part I.

First proof. Let $H$ denote $l$-adic cohomology for a prime $l \nmid q$ : since by [29, Cor. 1 and Remark 2)] $p$ lifts as a projector $\tilde{p}$ in $\mathcal{M}_{H}$, we may compute this trace after applying $H$ to $\tilde{p}$ (we then have to consider $H(X)$ as a $\mathbf{Z} / 2$-graded vector space and compute a super-trace.) Let $H(X)=V \oplus W$, with $V=\operatorname{Ker}(H(\tilde{p})-1)$ 
and $W=\operatorname{Ker}(H(\tilde{p}))$. View $F_{X}$ as a homological correspondence. Since it is central, it commutes with $\tilde{p}$, hence $H\left(F_{X}\right)$ respects $V$ and $W$ and

$$
\begin{aligned}
\operatorname{tr}\left(\tilde{p} F_{X}\right)=\operatorname{tr}\left(H\left(\tilde{p} F_{X}\right)\right)=\operatorname{tr}\left(H\left(\left(\tilde{p} F_{X}\right)_{\mid V}\right)+\operatorname{tr}\left(H\left(\left(\tilde{p} F_{X}\right)_{\mid W}\right)\right.\right. & \\
& =\operatorname{tr}\left(H\left(F_{X}\right)_{\mid V}\right) .
\end{aligned}
$$

Since the minimum polynomial of $H\left(F_{X}\right)$ kills $H\left(\left(F_{X}\right)_{\mid V}\right)$, the eigenvalues of the latter are algebraic integers [SGA7, Exp. XXI, Th. 5.2 .2 p. 386]. Hence $\operatorname{tr}\left(\tilde{p} F_{X}\right)$ is an algebraic integer and therefore is in $\mathbf{Z}$.

Second proof. Let $A=\operatorname{End}(h(X))$ : it is a semi-simple Q-algebra. By Proposition 1.2, there exists a central invertible element $\mu \in A$ such that $\operatorname{tr}(f)=$ $\operatorname{Trd}_{A}(\mu f)$ for any $f \in A$, where $\operatorname{Trd}_{A}$ is the reduced trace of $A$ (see Definition 1.1). By Theorem 5.6, the existence of a Weil cohomology theory implies that the components of $\mu$ on the simple factors $A_{i}$ of $A$ are integers. It is therefore sufficient to show that, for any $i$, the rational number $\operatorname{Trd}_{A_{i}}\left(e_{i} p F_{X}\right)$ is an integer, where $e_{i}$ is the central idempotent projecting to $A_{i}$.

Let $Z_{i}$ be the centre of $A_{i}$. Since $e_{i} F_{X} \in Z_{i}$, we have

$$
\operatorname{Trd}_{A_{i}}\left(e_{i} p F_{X}\right)=\operatorname{Tr}_{Z_{i} / \mathbf{Q}}\left(e_{i} F_{X} \operatorname{Trd}_{A_{i} / Z_{i}}(p)\right)
$$

Since $p$ is an idempotent, $\operatorname{Trd}_{A_{i} / Z_{i}}(p)$ is a positive integer (equal to the rank of the corresponding projector after extending scalars from $Z_{i}$ to $\overline{\mathbf{Q}}$ ). Therefore it suffices to prove that $\operatorname{Tr}_{Z_{i} / \mathbf{Q}}\left(e_{i} F_{X}\right)$ is an integer. But the minimal polynomial $P$ of $e_{i} F_{X}$ in $A_{i}$ divides the minimal polynomial $Q$ of $F_{X}$ in $A$ (because the projection $A \rightarrow A_{i}$ is a homomorphism of unital rings). Since $F_{X}$ is the graph of a morphism, it lies in the subalgebra of integral correspondences modulo numerical equivalence, which is a finitely generated $\mathbf{Z}$-module. Thus $Q$ is monic with integer coefficients, and so is $P$.

8.2. Remark. Note that the first proof of Theorem 8.1 uses [29, Remark 2 p. 451] which relies on Katz-Messing [38], hence ultimately on Deligne's proof of the Riemann hypothesis. This proof uses also Deligne's integrality theorem of SGA7, hence relies on two deep arithmetic results. The second proof uses Jannsen's semi-simplicity theorem [29, Th. 1] and the integrality result of Theorem 5.6; both results only rely on the existence of a Weil cohomology theory. 


\section{Number of RAtional points modulo $q$}

9.1. Theorem. The homomorphism (8.1) induces a ring homomorphism

$$
\overline{\#}_{n}: K_{0}\left(\mathcal{M}_{\text {num }}^{\mathrm{o}}\right) \rightarrow \mathbf{Z} / q^{n} .
$$

This follows from Theorem 8.1 and Proposition $7.7 \mathrm{~d}$ ) (note that $\#_{n}(\mathbb{L})=$ $\left.q^{n}\right)$.

From this we deduce:

9.2. Corollary. For $X, Y, f$ as in Proposition 7.6, we have

$$
\left|X\left(\mathbf{F}_{q}\right)\right| \equiv\left|Y\left(\mathbf{F}_{q}\right)\right| \quad(\bmod q) .
$$

This corollary has the following special cases:

9.3. Corollary (Esnault [19]). Let $X$ be a smooth projective variety over $\mathbf{F}_{q}$ such that $C H_{0}\left(X_{\mathbf{F}_{q}(X)}\right) \otimes \mathbf{Q}=\mathbf{Q}$. Then $\left|X\left(\mathbf{F}_{q}\right)\right| \equiv 1(\bmod q)$.

Proof. This is the case $Y=\operatorname{Spec} \mathbf{F}_{q}$.

9.4. Corollary (Fakhruddin-Rajan [22, Th. 1.2]). Let $f: X \rightarrow Y$ be a dominant morphism of smooth projective varieties over $\mathbf{F}_{q}$. If the map $f_{*}: C H_{0}\left(X_{\overline{\mathbf{F}_{q}(X)}}\right) \rightarrow$ $C H_{0}\left(Y_{\overline{\mathbf{F}_{q}(X)}}\right)$ is an isomorphism, then $\left|X\left(\mathbf{F}_{q}\right)\right| \equiv\left|Y\left(\mathbf{F}_{q}\right)\right|(\bmod q)$.

9.5. Remark. Fahkruddin and Rajan actually prove this under the weaker hypothesis that $X$ and $Y$ are smooth proper. One could "catch" this generality by enlarging the category of pure motives.

Here is a variant. Let us say that two smooth projective varieties $X$ and $Y$ are rationally connected-equivalent if there exist separably rationally connected smooth projective varieties $Z$ and $T$ and dominant rational maps $X \times Z \rightarrow Y$, $Y \times T \rightarrow X$. If $X$ and $Y$ are stably birationally equivalent, they are rationally connected equivalent.

9.6. Corollary. The number of rational points modulo $q$ is a rational connected-equivalence invariant of smooth projective $\mathbf{F}_{q}$-varieties (in particular, a stable birational invariant). 
Proof. Let $X, Y, Z, T$ be as in the definition of rational connected equivalence, and let $f, g$ be the two dominant rational maps. By Remark $7.4, \bar{h}(Y)$ is a direct summand of $\bar{h}(X \times Z)$ and $\bar{h}(X)$ is a direct summand of $\bar{h}(Y \times T)$. By Example $7.3, \bar{h}(Z)=\bar{h}(T)=\mathbf{1} \in \mathcal{M}_{\text {rat }}^{\mathrm{o}}$. Since $\bar{h}(X \times Z)=\bar{h}(X) \otimes \bar{h}(Z)$ and $\bar{h}(Y \times T)=\bar{h}(Y) \otimes \bar{h}(T), \bar{h}(X)$ and $\bar{h}(Y)$ are direct summands of each other in $\mathcal{M}_{\text {rat. }}^{\mathrm{o}}$. By semi-simplicity, their images in $\mathcal{M}_{\text {num }}^{\mathrm{o}}$ are isomorphic, hence $\overline{\#}_{n}(\bar{h}(X))=\overline{\#}_{n}(\bar{h}(Y))$ for all $n \geq 1$.

The following corollary was inspired by a discussion with A. Chambert-Loir.

9.7. Corollary. Suppose that $X$ is birationally dominated by a product $Y \times A$, where $Y$ verifies the conditions of Corollary 9.3 and $A$ is a supersingular abelian variety. Then $\left|X\left(\mathbf{F}_{q}\right)\right| \equiv 1(\bmod \sqrt{q})$. In particular, $X(k) \neq \emptyset$.

Proof. The statement means that $\left|X\left(\mathbf{F}_{q}\right)\right|-1$ is divisible by $\sqrt{q}$ in the ring of integers of $\mathbf{Q}(\sqrt{q})$ (in particular, in $\mathbf{Z}$ if $q$ is a square). In $\mathcal{M}_{\text {num }}^{\text {eff }}$, write $h(A)=\bigoplus h^{i}(A)$ where multiplication by $n$ acts on $h^{i}(A)$ as $n^{i}$. By definition of supersingular, $A$ becomes isogenous to a power of a supersingular elliptic curve $E$ after a finite extension of scalars $k / \mathbf{F}_{q}$. Hence $h\left(A_{k}\right) \simeq h(E)^{\otimes g}(g=\operatorname{dim} A)$ in $\mathcal{M}_{\text {rat }}^{\text {eff }}(k)$. The eigenvalues of Frobenius for $E$ are of the form $\zeta \sqrt{q^{\prime}}$ with $q^{\prime}=|k|$ and $\zeta$ a root of unity; by Tate's (Deuring's) theorem on the endomorphisms of $E$, it follows that $h^{1}(E)^{\otimes 2} \simeq M \otimes \mathbb{L}$, where $M$ is an Artin motive. It then follows that $h\left(A_{k}\right)=\mathbf{1} \oplus h^{1}\left(A_{k}\right) \oplus N_{k} \otimes \mathbb{L}$ for some effective motive $N_{k} \in \mathcal{M}_{\text {rat }}^{\text {eff }}(k)$. By taking traces, we also get $h(A)=\mathbf{1} \oplus h^{1}(A) \oplus N \otimes \mathbb{L}$ for some effective motive $N \in \mathcal{M}_{\text {rat }}^{\text {eff }}\left(\mathbf{F}_{q}\right)$. Therefore, $\bar{h}(Y \times A) \simeq \bar{h}(A) \simeq \mathbf{1} \oplus \bar{h}^{1}(A)$ in $\mathcal{M}_{\text {num }}^{\text {o }}$. By Remark $7.81), \bar{h}(X)$ is a direct summand of this motive, and since it contains $\mathbf{1}$ it is of the form $\mathbf{1} \oplus \bar{h}^{1}(B)$ for some other supersingular abelian variety $B$. But $\#\left(\bar{h}^{1}(B)\right) \equiv 0$ $(\bmod \sqrt{q})$.

9.8. Remarks. 1) Using Remark 7.8 2), we may canonically associate to any function field $K / \mathbf{F}_{q}$ of a smooth projective variety a series of integers $\left(a_{n}\right)_{n \geq 1}$ such that, for all $n, \#_{n}(\bar{h}(K))=a_{n}\left(\bmod q^{n}\right)$ (see Remark 7.4 for the definition of $\bar{h}(K)$ ). Naturally $a_{n}$ need not be positive in general. More conceptually, we may associate to $K$ its zeta function, defined as the zeta function of the motive $i(\bar{h}(K))$.

2) Killing $\mathbb{L}^{\kappa}$ instead of $\mathbb{L}$ would yield congruences modulo $q^{\kappa}$ rather than modulo $q$, cf. $[19, \S 3]$; compare also [59]. But one would lose the fact that function fields 
have effective numerical motives as in the previous remarks.

3) Corollary 9.2 suggests the following question: let $f: X \rightarrow Y$ be a rational map between smooth projective varieties over $k=k_{0}(t)$, where $k_{0}$ is an algebraically closed field of characteristic 0 . Suppose that $f$ induces an isomorphism $X(K) / R \stackrel{\sim}{\longrightarrow} Y(K) / R$ for an algebraically closed field $K$ containing $k(X)$ and $k(Y)$, where $R$ denotes $R$-equivalence [10]. Is it true that $Y(k) \neq \emptyset \Rightarrow X(k) \neq \emptyset$ ? (The opposite implication, over any $k$ and without $R$-equivalence hypothesis, is a well-known theorem of Nishimura and Lang.)

Olivier Wittenberg has given a positive answer to this question, by reduction to the theorem of Graber-Harris-Starr [25].

\section{A COnjeCtural CONVERSE}

By Theorem 8.1, the functions $\#_{n}$ of (8.1) extend to ring homomorphisms $K_{0}\left(\mathcal{M}_{\text {num }}\right) \rightarrow \mathbf{Z}[1 / q]$, still denoted by $\#_{n}$.

10.1. Theorem. Assume that the Tate conjecture (for the order of the pole of the zeta function) holds. Let $M \in K_{0}\left(\mathcal{M}_{\text {num }}\right)$ be such that $\#_{n}(M) \in \mathbf{Z}$ for all $n \geq 1$. Then $M \in K_{0}\left(\mathcal{M}_{\text {num }}^{\text {eff }}\right)$.

Conversely, if this implication holds for any $M \in K_{0}\left(\mathcal{M}_{\text {num }}\right)$, then the Tate conjecture holds.

Proof. Write $M=\sum m_{i}\left[S_{i}\right]$, where $m_{i} \in \mathbf{Z} \backslash\{0\}$ and the $S_{i}$ are simple pairwise non-isomorphic motives. For each $i$, let $w_{i}$ be a Weil number of $S_{i}$, that is, a root of the minimum polynomial of $F_{S_{i}}$, and $K_{i}=\mathbf{Q}\left(w_{i}\right)$. Then $\#_{n}(M)=$ $\sum m_{i} \operatorname{tr}_{K_{i} / \mathbf{Q}}\left(w_{i}^{n}\right)$. It follows from the assumption and from [41, Lemma 2.8] that $w_{i}$ is an algebraic integer for all $i$. (To apply loc. cit., compute in a Galois extension of $\mathbf{Q}$ containing all $K_{i}$ and observe that the Tate conjecture implies that no $w_{i}$ is equal to a conjugate of $w_{j}$ for $i \neq j$ [46, proof of Prop. 2.6].)

By Honda's theorem, for each $i$ there is an abelian variety $A_{i}$ over $\mathbf{F}_{q}$ and a simple direct summand $T_{i}$ of $h\left(A_{i}\right)$ whose Weil numbers are the Galois orbit of $w_{i}$ (ibid.). Reapplying Tate's conjecture, we get that $S_{i} \simeq T_{i}$, hence $S_{i}$ is effective for all $i$.

To prove the converse, let $M \in \mathcal{M}_{\text {num }}$ be simple and such that $F_{M}=1$. Then $\#_{n}(M)=1$ for all $n$. Therefore $M$ is effective. Writing $M$ as $(X, p)$ for some smooth projective variety $X$, we have that $M$ is a direct summand of $h^{0}(X)$ for 
weight reasons. It follows easily that $M \simeq 1$. By [24, Th. 2.7] (see also Theorem 17.2), this implies the Tate conjecture.

10.2. Corollary. The ring homomorphism

$$
\left(\overline{\#}_{n}\right)_{n \geq 1}: K_{0}\left(\mathcal{M}_{\text {num }}^{\mathrm{o}}\right) \rightarrow \prod_{n=1}^{\infty} \mathbf{Z} / q^{n}
$$

is injective if and only if the Tate conjecture is true.

Proof. It suffices to show that this injectivity is equivalent to the effectivity condition of Theorem 10.1, which is easy by semi-simplicity.

10.3. Remarks. 1) Unfortunately we have to apply the Tate conjecture to $S_{i} \otimes T_{i}^{*}$ in the proof of Theorem 10.1, hence cannot provide a hypothesis only involving $M$.

2) This argument may easily be modified to show that the Tate conjecture implies the generalised Tate conjecture, which says that if $M \in \mathcal{M}_{\text {hom }}$ is such that $H_{l}(M)$ is effective (in the sense that all Frobenius eigenvalues $w_{i}$ are algebraic integers), then $M$ is effective. Indeed, for such $M$, the Tate conjecture implies that $H_{l}(M)$ is semi-simple. To every (conjugacy class of) $w_{i}$, associate a pair $\left(A_{i}, T_{i}\right)$ as in the proof of Theorem 10.1. Then $H_{l}(M) \simeq \bigoplus H_{l}\left(T_{i}\right)$ as Galois modules, and $M \simeq \bigoplus T_{i}$ by the Tate conjecture.

3) Fontaine asked if one could describe the image of the homomorphism in Corollary 10.2. Here is an easy partial answer: for any $M \in \mathcal{M}_{\text {num }}^{\text {eff }}$, the sequence $\#_{n}(M)$ satisfies a recurrence relation, given by the minimal polynomial of $F_{M}$. (In particular, the image definitely does not lie in the inverse limit of the $\mathbf{Z} / q^{n}$.)

\section{Part III. Motivic zeta functions of motives}

\section{INTRODUCTION}

Let $\mathcal{M}$ be a tensor category with coefficients in a field $K$ of characteristic 0 , that is, a $K$-linear pseudo-abelian symmetric monoidal category such that the tensor product $\otimes$ of $\mathcal{M}$ is bilinear. Then symmetric and exterior powers of an object $M \in \mathcal{M}$ make sense, by using appropriate projectors relative to the action of the symmetric groups on tensor powers of $M$. One may therefore introduce 
the zeta function of $M$, a power series with coefficients in $K_{0}(\mathcal{M})[2, \S 13.3 .2]$ :

$$
Z_{\mathcal{M}}(M, T)=\sum_{n \geq 0}\left[S^{n}(M)\right] T^{n}
$$

Yves André showed that this zeta function is rational when $\mathcal{M}=\mathcal{M}(k)$ is the category of pure motives for some adequate equivalence relation over a field $k$ and $M$ is finite-dimensional in the sense of Kimura-O'Sullivan (ibid., Prop. 13.3.3.1). He raised the question of functional equations, in the light of Kapranov's result for curves [37]; this was achieved by Franziska Heinloth in [26] when $M$ is the motive of an abelian variety. Heinloth also proved in [26, Lemma 4.1] that André's rationality argument in fact applies to any finite-dimensional object in any $K$ linear tensor category $\mathcal{M}$.

In the same vein, we propose in Theorem 12.1 below a functional equation for any finite-dimensional $M \in \mathcal{M}$ in any rigid $K$-linear tensor category; this theorem has a very simple proof. In particular, modulo homological equivalence we get a functional equation for the motivic zeta function of any smooth projective variety $X$ for which the sign conjecture holds, that is, the sum of the even Künneth projectors of $X$ is algebraic ( $c f$. Definition 5.8). For instance, this functional equation holds for any $X$ over a finite field, or for surfaces over any field.

The main difference with Heinloth's formula is that ours involves the determinant of $M$, an invertible object of $\mathcal{M}$ to be defined in Proposition 11.3. This idea already appears in André's book [2, Proof of 12.1.6.3]. For this, we use the theory of 1-dimensional objects developed by Kimura in [40]. In the case of motives, we conjecture that $\operatorname{det}(M)$ is always the tensor product of a power of the Lefschetz motive and an Artin motive of square 1: we show that this conjecture implies the conservativity of realisation functors on finite-dimensional motives, follows from the Hodge or the Tate conjecture (see Proposition 14.2) and is true in important special cases (see Corollary 14.5 and Remark $14.6 \mathrm{~d}$ )).

Theorem 12.1 almost provides a cycle-theoretic proof of the functional equation for the usual zeta functions of motives over finite fields, see Remark 15.6 3). The catch is the sign conjecture. Unfortunately, the only known proof of this conjecture over a finite field is by Katz-Messing [38], who rely on Deligne's 
proof of the Riemann hypothesis [11]... One could use Theorem 12.1 in the category of $l$-adic sheaves, but this is not much more than the original approach of Grothendieck et al. ${ }^{6}$

Over general fields, the situation is even more open. Nevertheless the class of smooth projective varieties for which the sign conjecture is known is quite respectable: it contains varieties of dimension $\leq 2$, abelian varieties and complete intersections in a projective space. It is also stable under products. This puts the remark of $[26, \S 6]$ in a broader context.

\section{The Determinant}

Let $\mathcal{M}$ be a $K$-linear tensor category, where $K$ is a field of characteristic 0 . We assume that $\mathcal{M}$ is rigid and that $\operatorname{End}_{\mathcal{M}}(\mathbf{1})=K$. For any $M$, we write $\chi(M)=$ $\operatorname{tr}\left(1_{M}\right) \in K$; this is the Euler characteristic of $M$. Unifying the terminologies of Kimura and O'Sullivan, we shall say that $M$ is positive (resp. negative) if there exists an integer $N>0$ such that $\Lambda^{N}(M)=0$ (resp. $\left.S^{N}(M)=0\right)$, and finitedimensional if $M \simeq M^{+} \oplus M^{-}$where $M^{+}$is positive and $M^{-}$is negative. It can then be shown that in the positive (resp. negative) case, $\chi(M)$ is a nonnegative (resp. nonpositive) integer and that the smallest $N$ as above is $\chi(M)+1$ (resp. $-\chi(M)+1)([39],[4,9.1 .7])$.

We denote by $\mathcal{M}_{\text {kim }}$ the full subcategory of finite-dimensional objects in $\mathcal{M}$ : it is thick and rigid ([39], [4, 9.1.4 and 9.1.12]).

Recall that if $M$ is finite-dimensional, a decomposition $M \simeq M^{+} \oplus M^{-}$is unique up to isomorphism $([39],[4,9.1 .10])$. This allows us to set

$$
\chi^{+}(M)=\chi\left(M^{+}\right), \quad \chi^{-}(M)=\chi\left(M^{-}\right) .
$$

11.1. Definition ([40, 15.2.3 and 15.2.4]). An object $L \in \mathcal{M}$ is

- invertible if there exists an object $T$ and an isomorphism $L \otimes T \simeq \mathbf{1}$;

- 1-dimensional if it is either positive or negative, and $\chi(M)=1$ ( $M$ positive) or $\chi(M)=-1$ ( $M$ negative).

\footnotetext{
${ }^{6} \overline{\text { André pointed }}$ out that one could also use his category of motives for motivated cycles [1].
} 
We denote by $\operatorname{Pic}(\mathcal{M})$ the full subcategory of $\mathcal{M}$ consisting of invertible objects: it is a symmetric monoidal subcategory of $\mathcal{M}$. Similarly to [44], we also define

$$
\operatorname{Pic}^{*}(\mathcal{M})=\operatorname{Pic}(\mathcal{M}) \times\{ \pm 1\}
$$

with

$$
\operatorname{Pic}^{*}(\mathcal{M})\left((L, \varepsilon),\left(L^{\prime}, \varepsilon^{\prime}\right)\right)= \begin{cases}\operatorname{Pic}(\mathcal{M})\left(L, L^{\prime}\right) & \text { if } \varepsilon=\varepsilon^{\prime} \\ \emptyset & \text { if } \varepsilon \neq \varepsilon^{\prime} .\end{cases}
$$

This is a symmetric monoidal category for the rule

$$
(L, \varepsilon) \otimes\left(L^{\prime}, \varepsilon^{\prime}\right)=\left(L \otimes L^{\prime}, \varepsilon \varepsilon^{\prime}\right)
$$

with the commutativity constraint

$$
\left(L,(-1)^{e}\right) \otimes\left(L^{\prime},(-1)^{e^{\prime}}\right) \rightarrow\left(L^{\prime},(-1)^{e^{\prime}}\right) \otimes\left(L,(-1)^{e}\right)
$$

given by $(-1)^{e e^{\prime}} R_{L, L^{\prime}}$, where $R$ is the commutativity constraint of $\otimes$ in $\mathcal{M}$ (restricted to $\operatorname{Pic}(\mathcal{M}))$.

11.2. Lemma. a) An object $L \in \mathcal{M}$ is invertible if and only if it is 1-dimensional. Moreover, $L$ is positive (resp. negative) if and only if the switch of $L \otimes L$ is trivial (resp. equals -1).

b) If $M$ is positive, $\Lambda^{\chi(M)}(M)$ is invertible.

c) If $M$ is negative, $S^{-\chi(M)}(M)$ is invertible.

Proof. a) is the contents of [40, 15.2.6 and 15.2.9] (for the last statement, see the proof of 15.2 .9 in loc. cit.). In b) and c), it then suffices to prove that these objects are 1-dimensional. In case b), by [4, 7.2.4], $\chi\left(\Lambda^{n}(M)\right)=\left(\begin{array}{c}\chi(M) \\ n\end{array}\right)$, and in case c) $\chi\left(S^{n}(M)\right)=\left(\begin{array}{c}\chi(M)+n-1 \\ n\end{array}\right)$ by the same reference.

11.3. Proposition (compare [40, 15.4.3]). Let $\mathcal{M}_{\text {kim }}$ be the full subcategory of $\mathcal{M}$ formed of finite dimensional objects and $\mathcal{M}_{\text {kim }}^{\text {is }}$ the subcategory of $\mathcal{M}_{\text {kim }}$ with the same objects but morphisms restricted to isomorphisms. We view $\mathcal{M}_{\mathrm{kim}}^{\mathrm{is}}$ as a symmetric monoidal category with respect to the direct sum. Then there exist monoidal functors

$$
\begin{array}{r}
\operatorname{det}: \mathcal{M}_{\text {kim }}^{\text {is }} \rightarrow \operatorname{Pic}(\mathcal{M}) \\
\underline{\operatorname{det}: \mathcal{M}_{\text {kim }}^{\text {is }} \rightarrow \operatorname{Pic}^{*}(\mathcal{M})}
\end{array}
$$

such that

$$
\operatorname{det}(M)=\Lambda^{\chi^{+}(M)}\left(M^{+}\right) \otimes S^{-\chi^{-}(M)}\left(M^{-}\right)^{*}
$$


if $M \simeq M^{+} \oplus M^{-}$(cf. (11.1)), and

$$
\underline{\operatorname{det}}(M)=\left(\operatorname{det}(M),(-1)^{\chi^{+}(M)}\right) \text {. }
$$

These functors are well-determined up to unique isomorphisms.

Proof. To define det correctly, we fix for each $M \in \mathcal{M}_{\text {kim }}$ a decomposition $M \simeq$ $M^{+} \oplus M^{-}$and use the formula in the proposition. Let $N \simeq N^{+} \oplus N^{-}$and $f: M \rightarrow N$ be an isomorphism. To $\operatorname{define} \operatorname{det}(f): \operatorname{det}(M) \rightarrow \operatorname{det}(N)$, we note that $\mathcal{M}\left(L, L^{\prime}\right) \stackrel{\sim}{\longrightarrow} \overline{\mathcal{M}}\left(\bar{L}, \bar{L}^{\prime}\right)$ for $L \simeq L^{\prime} \in \operatorname{Pic}(\mathcal{M})$, where $\overline{\mathcal{M}}=\mathcal{M} / \mathcal{N}$ (here $\mathcal{N}$ is the ideal of morphisms universally of trace 0 defined in (1.2)): indeed, this set is then a 1-dimensional vector space over $K=\operatorname{End}_{\mathcal{M}}(\mathbf{1}) \simeq \operatorname{End}_{\mathcal{M}}(L)$. By $[4,9.2 .1 \mathrm{a})], \bar{f}: \bar{M} \rightarrow \bar{N}$ may be written as $\bar{f}^{+} \oplus \bar{f}^{-}$, and we $\operatorname{define} \operatorname{det}(f)$ as $\operatorname{det}\left(\bar{f}^{+}\right) \otimes \operatorname{det}\left(\bar{f}^{-}\right)^{*}$, where $\operatorname{det}\left(\bar{f}^{+}\right)$and $\operatorname{det}\left(\bar{f}^{-}\right)$are defined via the exterior and symmetric powers. This clearly defines a functor, which shows that det is well-defined up to unique isomorphism. A monoidal structure on det is defined by the composition

$$
\begin{aligned}
& \operatorname{det}(M) \otimes \operatorname{det}(N)= \\
& \quad \Lambda^{\chi^{+}(M)}\left(M^{+}\right) \otimes S^{-\chi^{-}(M)}\left(M^{-}\right)^{*} \otimes \Lambda^{\chi^{+}(N)}\left(N^{+}\right) \otimes S^{-\chi^{-}(N)}\left(N^{-}\right)^{*} \\
& \stackrel{\sim}{\longrightarrow} \Lambda^{\chi^{+}(M)}\left(M^{+}\right) \otimes \Lambda^{\chi^{+}(N)}\left(N^{+}\right) \otimes S^{-\chi^{-}(M)}\left(M^{-}\right)^{*} \otimes S^{-\chi^{-}(N)}\left(N^{-}\right)^{*} \\
& \stackrel{\sim}{\longrightarrow} \Lambda^{\chi^{+}(M)+\chi^{+}(N)}\left(M^{+} \oplus N^{+}\right) \otimes S^{-\chi^{-}(M)-\chi^{-}(N)}\left(M^{-} \oplus N^{-}\right)^{*} \\
& \\
& \quad \operatorname{det}(M \oplus N)
\end{aligned}
$$

where the first isomorphism is the switch and the second is the obvious one. A monoidal structure on $\underline{\text { det }}$ is then defined by the composition

$$
\begin{aligned}
\underline{\operatorname{det}}(M) \otimes \underline{\operatorname{det}}(N)=(\operatorname{det}(M) & \left.\otimes \operatorname{det}(N),(-1)^{\chi^{+}(M)}(-1)^{\chi^{+}(N)}\right) \\
& \stackrel{\sim}{\longrightarrow}\left(\operatorname{det}(M \oplus N),(-1)^{\chi^{+}(M \oplus N)}\right)=\underline{\operatorname{det}}(M \oplus N)
\end{aligned}
$$

where the isomorphism comes from the monoidal structure on det.

11.4. Remarks. 1) If $L$ is invertible, an object $T$ as in Definition 11.1 is unique up to unique isomorphism, and is given by the dual $L^{*}$ of $L$.

2) If $M$ is positive, $\operatorname{det}(M)$ is positive. If $M$ is negative, $\operatorname{det}(M)$ has the sign $(-1)^{\chi(M)}$ : this follows from the proof of Lemma $\left.11.2 \mathrm{c}\right)$. 
3) There is a competing functor to det, which is more natural:

$$
\underline{\operatorname{det}}^{\prime}(M)=\left(\operatorname{det}(M),(-1)^{\chi(M)}\right) .
$$

If $\mathcal{A}$ admits a (symmetric) $\otimes$-functor to a category of $\mathbf{Z} / 2$-graded vector spaces, then the computations of [44] imply that det $^{\prime}$ is symmetric monoidal; I suppose that this can be proven in general, but I haven't found a good proof. In any case, this shows that det is not symmetric in general. Nevertheless, this is the object which will control the functional equation in the next section.

The following lemma slightly extends Proposition 11.3, and will be used in the proof of Proposition 14.2.

11.5. Lemma. Let $f: M_{1} \rightarrow M_{2}$ be a morphism between finite-dimensional objects such that $\chi^{+}\left(M_{1}\right)=\chi^{+}\left(M_{2}\right)$ and $\chi^{-}\left(M_{1}\right)=\chi^{-}\left(M_{2}\right)$. Then $f$ induces a canonical morphism $\operatorname{det}(f): \operatorname{det}\left(M_{1}\right) \rightarrow \operatorname{det}\left(M_{2}\right)$ in $\mathcal{M} / \mathcal{N}$. Moreover, $f$ is an isomorphism if and only if $\operatorname{det}(f)$ is.

Proof. The construction of $\operatorname{det}(f)$ is the same as in the proof of Proposition 11.3. It remains to see that, if $\operatorname{det}(f)$ is an isomorphism, $f$ is an isomorphism. For this, we work in $\mathcal{M}_{\text {kim }}$. By $[4,9.2 .2], \mathcal{M}_{\text {kim }} / \mathcal{N}$ is abelian semi-simple. Let $\bar{f}$ be the image of $f$ in this category: we may write $\bar{f}$ as the direct sum of an isomorphism $f^{\prime}: M_{1}^{\prime} \rightarrow M_{2}^{\prime}$ and a 0 morphism $f^{\prime \prime}: M_{1}^{\prime \prime} \rightarrow M_{2}^{\prime \prime}$. Then $\operatorname{det}(f)=$ $\operatorname{det}\left(f^{\prime}\right) \otimes \operatorname{det}\left(f^{\prime \prime}\right)$. But if $M_{1}^{\prime \prime}$ and $M_{2}^{\prime \prime}$ were nonzero, we would have $\operatorname{det}\left(f^{\prime \prime}\right)=0$, hence $\operatorname{det}(\bar{f})=0$, contrary to the hypothesis. Thus $\bar{f}$ is an isomorphism, and by the local nilpotency of $\mathcal{N}[4,9.1 .14], f$ is an isomorphism.

11.6. Lemma. a) If $M$ is positive, we have isomorphisms

$$
\Lambda^{n}\left(M^{*}\right) \simeq \Lambda^{\chi(M)-n}(M) \otimes \operatorname{det}(M)^{*}
$$

for all $n \in[0, \chi(M)]$.

b) If $M$ is negative, we have isomorphisms

$$
S^{n}\left(M^{*}\right) \simeq S^{-\chi(M)-n}(M) \otimes \operatorname{det}(M)^{*}
$$

for all $n \in[0,-\chi(M)]$.

Proof. a) Note that $\Lambda^{n}\left(M^{*}\right) \simeq \Lambda^{n}(M)^{*}$. The obvious pairing

$$
\Lambda^{n}(M) \otimes \Lambda^{\chi(M)-n}(M) \rightarrow \operatorname{det}(M)
$$


and its dual applied with $M^{*}$ instead of $M$ define morphisms

$$
\begin{aligned}
& \Lambda^{n}(M) \otimes\left(\Lambda^{\chi(M)-n}(M) \otimes \operatorname{det}(M)^{*}\right) \rightarrow \mathbf{1} \\
& \mathbf{1} \rightarrow\left(\Lambda^{\chi(M)-n}(M) \otimes \operatorname{det}(M)^{*}\right) \otimes \Lambda^{n}(M)
\end{aligned}
$$

and it is easy to check that they verify the duality axioms [17]. The proof for b) is similar.

\section{The FunCtional Equation}

Define a multiplicative homomorphism

$$
\text { [] }: K_{0}\left(\operatorname{Pic}^{*}(\mathcal{M})\right) \rightarrow K_{0}(\mathcal{M})
$$

by $[(L, \varepsilon)]=\varepsilon[L]$. We can now state our theorem:

12.1. Theorem. Let $M \simeq M^{+} \oplus M^{-}$be finite dimensional, and $M^{*}$ be its dual. Then we have:

$$
\begin{aligned}
Z_{\mathcal{M}}\left(M^{*}, T^{-1}\right)=[\underline{\operatorname{det}}(M)] T^{\chi(M)} Z_{\mathcal{M}} & (M, T) \\
& =(-1)^{\chi^{+}(M)}[\operatorname{det}(M)] T^{\chi(M)} Z_{\mathcal{M}}(M, T) .
\end{aligned}
$$

Proof. Since the zeta function transforms direct sums into products, we may treat separately the cases where $M$ is positive and negative. Note that $M^{*}$ has the same sign as $M$.

1. If $M$ is positive, we have (see [26, Lemma 4.1]):

$$
Z_{\mathcal{M}}\left(M^{*}, T^{-1}\right)^{-1}=\sum_{n=0}^{\chi(M)}\left[\Lambda^{n}\left(M^{*}\right)\right](-T)^{-n} .
$$


Applying Lemma 11.6 a), we may rewrite this sum as

$$
\begin{gathered}
{\left[\operatorname{det}(M)^{*}\right] \sum_{n=0}^{\chi(M)}\left[\Lambda^{\chi(M)-n}(M)\right](-T)^{-n}} \\
=\left[\operatorname{det}(M)^{*}\right] \sum_{n=0}^{\chi(M)}\left[\Lambda^{n}(M)\right](-T)^{n-\chi(M)} \\
=\left[\operatorname{det}(M)^{*}\right](-T)^{-\chi(M)} \sum_{n=0}^{\chi(M)}\left[\Lambda^{n}(M)\right](-T)^{n} \\
=\left[\operatorname{det}(M)^{*}\right](-T)^{-\chi(M)} Z_{\mathcal{M}}(M, T)^{-1} .
\end{gathered}
$$

2. If $M$ is negative, we have

$$
Z_{\mathcal{M}}\left(M^{*}, T^{-1}\right)=\sum_{n=0}^{-\chi(M)} S^{n}\left(M^{*}\right) T^{-n}
$$

Applying Lemma 11.6 b), we may rewrite this sum as

$$
\begin{aligned}
& {\left[\operatorname{det}(M)^{*}\right] \sum_{n=0}^{-\chi(M)}\left[S^{-\chi(M)-n}(M)\right] T^{-n} } \\
&= {\left[\operatorname{det}(M)^{*}\right] \sum_{n=0}^{-\chi(M)}\left[S^{n}(M)\right] T^{n+\chi(M)} } \\
&\left.=\left[\operatorname{det}(M)^{*}\right] T^{\chi(M)} \sum_{n \geq 0} S^{n}(M)\right] T^{n} \\
&=\left[\operatorname{det}(M)^{*}\right] T^{\chi(M)} Z_{\mathcal{M}}(M, T) .
\end{aligned}
$$

12.2. Remarks. a) As remarked in $[2,13.2 .1 .1]$, the map $K_{0}\left(\mathcal{M}_{\text {kim }}\right) \rightarrow K_{0}\left(\mathcal{M}_{\text {kim }} / \mathcal{N}\right)$ is an isomorphism thanks to the local nilpotency of $\mathcal{N}$ : therefore it makes no difference to work in $\mathcal{M}_{\text {kim }}$ or in $\mathcal{M}_{\text {kim }} / \mathcal{N}$ as long as the zeta function is concerned. b) One may wonder if $\chi^{-}(M)$ is always even: this would give a nicer formula in Theorem 12.1. As Yves André pointed out, this is of course false if we take for $\mathcal{M}$ the category of $\mathbf{Z} / 2$-graded $\mathbf{Q}$-vector spaces. However, this turns out to be true (most of the time, and conjecturally always) if $\mathcal{M}$ is the category of motives with rational coefficients over a field: André gave us an argument for this in 
characteristic 0 , and his argument inspired part of the proofs given in the next section.

\section{The CASE OF MOTIVES}

We now assume that $\mathcal{M}=\mathcal{M}_{\sim}(k)$ is the category of motives over a field $k$ with respect to a given adequate equivalence relation $\sim$ : we shall often abbreviate $\mathcal{M}_{\sim}(k)$ into $\mathcal{M}(k)$. The two main examples for $\sim$ are rational and homological equivalence. We only work with finite-dimensional motives $M$ relatively to $\sim$. If $\sim$ is homological equivalence, this hypothesis is equivalent to the sign conjecture (algebraicity of the even Künneth projector), $c f$. Lemma 5.9.

Notice in any case that, in view of Remark 12.2 a), the motivic zeta function of $M$ with respect to $\sim$ is the same as its motivic zeta function with respect to any coarser adequate equivalence relation. Similarly, if $H$ is a classical Weil cohomology theory (in the sense of [2, 3.4]) and $\sim$ is coarser than $H$-equivalence, then $K_{0}\left(\mathcal{M}_{H}(k)\right) \stackrel{\sim}{\longrightarrow} K_{0}\left(\mathcal{M}_{\sim}(k)\right) \stackrel{\sim}{\longrightarrow} K_{0}\left(\mathcal{M}_{\text {num }}(k)\right)$ by the nilpotence result of [3, Prop. 5], without even restricting to finite-dimensional motives. Another such example is Voevodsky's nilpotence theorem [58], which implies that $K_{0}\left(\mathcal{M}_{\text {rat }}(k)\right) \stackrel{\sim}{\longrightarrow} K_{0}\left(\mathcal{M}_{\text {alg }}(k)\right)$. Under the conjecture of KimuraO'Sullivan that every Chow motive is finite-dimensional, or under the BlochBeilinson-Murre conjecture [30], $\mathcal{N}$ is locally nilpotent in $\mathcal{M}_{\text {rat }}(k)$, which would imply that $K_{0}\left(\mathcal{M}_{\sim}(k)\right)$ is independent of the given adequate equivalence relation $\sim$.

Let $L \in \mathcal{M}_{\sim}(k)$ be an invertible motive. Let $L_{\text {num }}$ be the image of $L$ in $\mathcal{M}_{\text {num }}(k)$. By [3, Prop. 5], we may lift $L_{\text {num }}$ to an invertible motive $L_{H} \in$ $\mathcal{M}_{H}(k)$ with respect to any classical Weil cohomology theory $H$, uniquely up to isomorphism. Then there is a unique integer $n \in \mathbf{Z}$ such that $H^{n}\left(L_{H}\right) \neq 0$.

13.1. Definition. The integer $n$ is called the weight of $L$.

That the weight does not depend on the choice of the classical cohomology theory follows from Artin's comparison theorem in characteristic 0 and from Deligne's proof of the Riemann hypothesis in characteristic $p$ [38].

13.2. Conjecture. Let $k$ be a field. There is no invertible motive $L \in \mathcal{M}(k)$ of odd weight $n$. 
Equivalently: there is no negative invertible motive in $\mathcal{M}(k)$.

In fact, we can almost prove Conjecture 13.2:

13.3. Proposition. Conjecture 13.2 is true in the following cases:

(1) $\operatorname{char} k=0$.

(2) $k$ is a regular extension of $\mathbf{F}_{q}$, where $q$ is not a square.

(3) $n=1$ and $L$ is effective.

Moreover, the Tate conjecture over finite fields implies Conjecture 13.2 in the remaining cases. (Can one avoid the recourse to the Tate conjecture???)

Proof. Clearly, we may work modulo homological equivalence.

(1) This is obvious via the Hodge realisation: in fact, any invertible pure Hodge structure is of the form $\mathbf{Q}(r)$ since the Hodge numbers go by symmetric pairs. (This was part of André's argument alluded to in Remark 12.2.) Alternately, we may use $l$-adic cohomology as follows: let $k_{0} \subseteq k$ be a finitely generated field over which the invertible motive $L$ is defined. Let $S$ be a regular model of $k_{0}$ of finite type over $\mathbf{Z}$. Then one may find a closed point $s \in S$ with residue field $\mathbf{F}_{p}$ ( $p$ a large enough prime number) such that $L$ has good reduction at $s .{ }^{7}$ This reduces us to the case where $k=\mathbf{F}_{p}$. Up to twisting $L$ by a power of $\mathbb{L}$, we may assume $L$ effective. Let $F$ be the Frobenius endomorphism of $L$. Since $\operatorname{End}(\mathbf{1})=\mathbf{Q}, \operatorname{tr}(F)$ is a rational number, which is also the (unique) eigenvalue of the action of $F$ on $H_{l}(L)$. But if $n$ is the weight of $L, H_{l}(L)$ is a direct summand of $H^{n}\left(\bar{X}, \mathbf{Q}_{l}\right)$ for some smooth projective variety $X$; by the Weil conjecture, $|\operatorname{tr}(F)|=p^{n / 2}$, hence $n$ is even.

(2) The same $l$-adic argument as in (1) works.

(3) Write $L$ as a direct summand of $h(X)$ for some smooth projective variety $X$. We may write $L$ as a direct summand of $h^{1}(X)$. (Note that there is always a canonical decomposition $h(X)=h^{0}(X) \oplus h^{1}(X) \oplus h^{\geq 2}(X)$ and that $\operatorname{Hom}\left(L, h^{0}(X)\right)=\operatorname{Hom}\left(L, h^{\geq 2}(X)\right)=0$ for weight reasons.) Replacing $X$ by its Albanese variety, we may assume that $X$ is an abelian variety $A$. Now, by

\footnotetext{
${ }^{7}$ Indeed, a closed point of the generic fibre of $S$ extends to a dominant 1-dimensional closed Zsubscheme $Z$ of $S$; a suitable open subset of $Z$ is regular, hence has a closed point as requested, by Čebotarev's density theorem.
} 
Poincaré's complete irreducibility theorem, the full subcategory of motives modulo homological equivalence consisting of the $h^{1}(A)$ is abelian semi-simple; thus $L=h^{1}(B)$ for some abelian variety $B$. But this is impossible, since $L$ is 1dimensional and $\operatorname{dim} h^{1}(B)$ has to be even.

Finally, if the Tate conjecture holds over $\mathbf{F}_{q^{2}}$, then the generalised Tate conjecture also holds thanks to Honda's theorem (compare Remark 10.32 )); hence, up to twisting $L$ by some power of the Lefschetz motive, we may assume that it is effective of weight 1 and we are reduced to (3).

13.4. Remark. Notice that Conjecture 13.2 becomes false over $\mathbf{F}_{q^{2}}$ after extending scalars from $\mathbf{Q}$ to a suitable quadratic extension, as shown by $h^{1}$ of a supersingular elliptic curve. Similarly, Conjecture 13.2 becomes (very) false over any field after extending scalars from $\mathbf{Q}$ to $\overline{\mathbf{Q}}$.

13.5. Theorem. a) If conjecture 13.2 is true for $k$ (compare Proposition 13.3), $\chi(M)$ is even for any negative $M \in \mathcal{M}(k)$. Thus, in this case, the functional equation of Theorem 12.1 simplifies as

$$
Z_{\mathcal{M}}\left(M^{*}, T^{-1}\right)=\operatorname{det}(M)(-T)^{\chi(M)} Z_{\mathcal{M}}(M, T) .
$$

b) $\chi^{-}(M)$ is even over any field $k$ if $M=h(X)$ for $X$ a smooth projective variety verifying the sign conjecture. We then have

$$
Z_{\mathcal{M}}\left(h(X), T^{-1}\right)=(-T)^{\chi(X)} \operatorname{det}(h(X)) Z_{\mathcal{M}}\left(h(X),[\mathbb{L}]^{-d} T\right) .
$$

This formula holds modulo homological equivalence, and even modulo rational equivalence if $h_{\mathrm{rat}}(X)$ is finite-dimensional.

Proof. a) If there exists a negative motive $M$ of odd Euler characteristic, then $\operatorname{det}(M)$ is negative of Euler characteristic -1 (see Remark 11.42 )). It remains to justify b): this is true since the odd-dimensional $l$-adic Betti numbers of $X$ are even by Hard Lefschetz [11, (4.1.5)]. The functional equation for $h(X)$ then follows from the formula $h(X)^{*}=h(X) \otimes \mathbb{L}^{-d}$, where $\mathbb{L}$ is the Lefschetz motive, and the obvious identity

$$
Z_{\mathcal{M}}\left(h(X) \otimes \mathbb{L}^{-d}, T\right)=Z_{\mathcal{M}}\left(h(X),[\mathbb{L}]^{-d} T\right)
$$




\section{The Determinant of A motive}

When $X$ is an abelian variety, (13.1) is Heinloth's formula, with the difference that the factor $\operatorname{det}(h(X))$ is replaced by 1 . We want to explain in this section why this happens. This is a good opportunity to introduce the following strengthening of Conjecture 13.2:

14.1. Conjecture. For any field $k, \operatorname{Pic}\left(\mathcal{M}_{\sim}(k)\right)$ is generated by $\mathbb{L}$ and Artin motives of square $\mathbf{1}$.

(Such Artin motives are in 1-to-1 correspondence with the elements of $k^{*} / k^{* 2}$.)

Recall that a functor $F$ is conservative if, when $F(f)$ is an isomorphism, $f$ is already an isomorphism. We shall say that $F$ is essentially injective if it is injective on isomorphism classes of objects. If $F$ is fully faithful, it is both conservative and essentially injective.

14.2. Proposition. a) Suppose that $\sim$ is finer than homological equivalence with respect to some Weil cohomology theory $H$. Then Conjecture 14.1 implies that $H$ is conservative on finite-dimensional motives.

b) If $k$ verifies Conjecture 13.2, the essential injectivity of the Hodge realisation (if char $k=0$ ) or the l-adic realisation over finitely generated fields implies Conjecture 14.1 for any $\sim$. In particular, Conjecture 14.1 follows from either the Hodge or the Tate conjecture.

Proof. a) Let $f: M_{1} \rightarrow M_{2}$ be a morphism between finite-dimensional motives such that $H(f)$ is an isomorphism. This implies that $\chi^{ \pm}\left(M_{1}\right)=\chi^{ \pm}\left(M_{2}\right)$, hence, by Lemma 11.5, $f$ induces a morphism $\operatorname{det}(f): \operatorname{det}(M) \rightarrow \operatorname{det}\left(M^{\prime}\right)$. Obviously, $H(\operatorname{det}(f))=\operatorname{det} H(f)$, hence $H(\operatorname{det}(f))$ is an isomorphism, and in particular $\operatorname{det}(f) \neq 0$. But Conjecture 14.1 easily implies that $\mathcal{M}\left(L, L^{\prime}\right)=0$ if $L \nsucceq L^{\prime}$, hence $\operatorname{det}(f)$ is an isomorphism and $f$ is an isomorphism by Lemma 11.5.

For b), we may reduce to the case where $\sim$ is finer than Hodge or $l$-adic equivalence, thanks to [3, Prop. 5].

1) Let us assume that char $k=0$ and that the Hodge realisation is conservative. Let $L$ be an invertible motive. We may assume that $L$ is defined over a subfield $k_{0}$ of C. As seen in the proof of Proposition 13.3, $H(L) \simeq H\left(\mathbb{L}^{n}\right)$ for some $n \in \mathbf{Z}$; by essential injectivity, $L_{\mathbf{C}} \simeq \mathbb{L}^{n}$, hence up to twisting we may assume 
that $L_{\mathbf{C}}=\mathbf{1}$. There is a finitely generated extension $k_{1}$ of $k_{0}$ such that $L_{k_{1}}=\mathbf{1}$, and after a suitable specialisation we may assume that $k_{1} / k_{0}$ is finite. Taking traces, we get that $L \otimes h\left(\operatorname{Spec} k_{1}\right) \simeq h\left(\operatorname{Spec} k_{1}\right)$ in $\mathcal{M}\left(k_{0}\right)$; hence $L \simeq L \otimes \mathbf{1}$ is a direct summand of $h\left(\operatorname{Spec} k_{1}\right)$ and is an Artin motive. But Artin motives are in 1-to-1 correspondence with rational Galois representations of $k$, and any rational character of a (pro)finite group has square 1 .

2) Let us assume that $k$ verifies Conjecture 13.2 and that the $l$-adic realisation is conservative on finitely generated subfields of $k$. Let $L$ be an invertible motive and let $R_{l}(L)$ be its $l$-adic realisation. Let $n$ be the weight of $L$, which is assumed to be even. As in the proof of Proposition 13.3 (1), we may assume $k$ finitely generated; using the same rationality argument as in this proof, we see that the eigenvalue of Frobenius acting on the specialisation of $R_{l}(L)$ at any finite place of good reduction and residue field $\mathbf{F}_{q}$ is of the form $\pm q^{n / 2}$. Thus Frobenius automorphisms act trivially on the $l$-adic representation $R_{l}\left(L^{\otimes 2} \otimes \mathbb{L}^{-n}\right)$; by Cebotarev's density theorem, it follows that $R_{l}\left(L^{\otimes 2} \otimes \mathbb{L}^{-n}\right)$ is trivial, hence $R_{l}\left(L \otimes \mathbb{L}^{-n / 2}\right)$ is given by a quadratic character of $k$. Let $A$ be the corresponding Artin motive of square 1: by essential injectivity we have $L \simeq A \otimes \mathbb{L}^{n / 2}$, as desired.

It remains to justify the last assertion of b). If the Hodge conjecture holds, then the Hodge realisation functor is essentially injective on the level of motives modulo homological equivalence. But let $L, L^{\prime} \in \operatorname{Pic}\left(\mathcal{M}_{\sim}(k)\right)$ : since $L$ and $L^{\prime}$ are finite-dimensional, Kimura's nilpotence theorem implies that any isomorphism between them modulo homological equivalence lifts to an isomorphism modulo $\sim$. The argument is the same with the Tate conjecture.

14.3. Remarks. 1) In $[2,12.1 .6 .3]$, André proves that the irreducibility of $\mathbf{1}$ implies the conservativity of a Weil cohomology $H$ with basically the same proof as the proof of Prop. 14.2 a) given above. It would be nice to clarify the relationship between this condition and Conjecture 14.1.

2) Conjecture 14.1 is obviously equivalent to the following: for any finite-dimensional $M \in \mathcal{M}(k)$, we have

$$
\operatorname{det}(M)=\mathbb{L}^{r} \otimes A
$$

for some $r \in \mathbf{Z}$, where $A$ is a 1-dimensional Artin motive of square $\mathbf{1}$. If $M$ is pure of weight $w$, it is easy to compute $r$ in terms of $w$ and $\chi(M)$ : as a direct 
summand of $M^{\otimes \chi(M)}$ or $\left(M^{*}\right)^{\otimes-\chi(M)}$ according to the parity of $w \operatorname{det}(M)$ is of weight $w \chi(M)$, hence we find $r=w \chi(M) / 2$.

It is interesting to investigate the reformulation of Remark 14.32 ) in the light of formal propreties of det, as follows:

14.4. Proposition. Let $\mathcal{M}$ be a rigid tensor $\mathbf{Q}$-linear category. Then, for any finite-dimensional $M, N$ :

(1) $\operatorname{det}(M \oplus N)=\operatorname{det}(M) \operatorname{det}(N)$.

(2) $\operatorname{det}(M \otimes N)=\operatorname{det}(M)^{\chi(N)} \otimes \operatorname{det}(N)^{\chi(M)}$.

(3) $\operatorname{det}\left(\Lambda^{n}(M)\right)=\operatorname{det}(M)^{r}$, with $r=n\left(\begin{array}{c}\chi(M) \\ n\end{array}\right) / \chi(M)$.

(4) $\operatorname{det}\left(S^{n}(M)\right)=\operatorname{det}(M)^{s}$, with $s=n\left(\begin{array}{c}\chi(M)+n-1 \\ n\end{array}\right) / \chi(M)$.

(5) $\operatorname{det}\left(M^{*}\right)=\operatorname{det}(M)^{*}$.

Proof. This follows readily from identities between Schur functors as in [26, §4].

14.5. Corollary. Let $\sim$ be homological equivalence.

a) The subset $K_{0}^{\prime}(\mathcal{M}(k))$ of $K_{0}(\mathcal{M}(k))$ consisting of differences of classes of finitedimensional motives that verify (14.1) is a sub- $\lambda$-ring of $K_{0}(\mathcal{M}(k))$.

b) Similarly, the subset $K_{0}^{\prime \prime}(\mathcal{M}(k))$ of $K_{0}(\mathcal{M}(k))$ consisting of differences of classes of finite-dimensional motives such that $A=1$ in (14.1) is a sub- $\lambda$-ring of $K_{0}(\mathcal{M}(k))$.

c) Suppose that $M$ is weakly polarisable in the sense that $M \simeq M^{*} \otimes \mathbb{L}^{n}$ for some integer $n$. Then $\operatorname{det}(M)^{2}=\mathbb{L}^{n \chi(M)}$.

d) Suppose that $X / k$ verifies the standard conjecture $B$ [43]. Then, for all $i \geq 0$, $\operatorname{det}\left(h^{i}(X)\right)^{2}=\mathbb{L}^{(-1)^{i} i b_{i}(X)}$, where $b_{i}(X)$ is the $i$-th Betti number of $X$. Moreover, letting $d=\operatorname{dim} X$ :

$$
\operatorname{det}(h(X))=\mathbb{L}^{\frac{d}{2}\left(\chi(X)-(-1)^{d} b_{d}(X)\right)} \otimes \operatorname{det}\left(h^{d}(X)\right) .
$$

e) $K_{0}^{\prime \prime}(\mathcal{M}(k))$ contains $\left[h^{1}(X)\right]$ for any smooth projective variety $X$. More precisely, $\operatorname{det}\left(h^{1}(X)\right) \simeq \mathbb{L}^{b_{1}(X) / 2}$.

f) If $C$ is a curve, $\operatorname{det}(h(C))=\mathbb{L}^{\chi(C) / 2}$.

g) If $A$ is an abelian variety, $\operatorname{det}(h(A))=1$.

Proof. a), b) and c) follow directly from Proposition 14.4 (see also Remark 11.4 1) for c)). In d), note first that the standard conjecture B implies the algebraicity 
of the Künneth projectors [43], which justifies the introduction of the $h^{i}(X)$; it also implies that all $h^{i}(X)$ verify the hypothesis of $\left.\mathrm{c}\right)$. The first claim of $\mathrm{d}$ ) then follows from c) (note that $\chi\left(h^{i}(X)\right)=(-1)^{i} b_{i}(X)$ ). We get the second one by grouping the terms $\operatorname{det}\left(h^{i}(X)\right)$ and $\operatorname{det}\left(h^{2 d-i}(X)\right)$ together for $i<d$, and using that $h^{2 d-i}(X) \simeq h^{i}(X)^{*} \otimes \mathbb{L}^{d}$ by Poincaré duality.

In e), note that $h^{1}(X) \simeq h^{1}(A)$, where $A$ is the Albanese variety of $X$; the claim then follows from the result of Shermenev [55] that $h^{i}(A) \simeq S^{i}\left(h^{1}(A)\right.$ ) (apply this for $i=2 \operatorname{dim} A$ ). f) follows from e). Finally, in g), $A$ verifies the standard conjecture $\mathrm{B}$ by Lieberman-Kleiman [41]; applying e), b) and the formula in Remark 14.32 ), we get $\operatorname{det}\left(h^{d}(A)\right)=\mathbb{L}^{d \chi\left(h^{d}(A)\right) / 2}$ for $d=\operatorname{dim} A$, and the claim of g) follows from d) by noting that $\chi(A)=0$.

14.6. Remarks. a) Theorem 13.5 and Corollary 14.5 f) give back Kapranov's functional equation for curves [37], with coefficients in $K_{0}(\mathcal{M}(k))$ (a little information is lost).

b) The reader familiar with Heinloth's paper will recognize part of her arguments in the proof of Corollary $14.5 \mathrm{~g}$ ). This corollary was actually inspired by reading her paper.

c) In view of Corollary $14.5 \mathrm{f}$ ) and g), one might expect that the Artin motive $A$ appearing in (14.1) is always $\mathbf{1}$ for $M$ of the form $h(X)$. This is wrong: rational surfaces give examples where (14.1) holds but $A$ is nontrivial. Indeed, for such a surface $S, h^{2}(S)=\mathbb{L} \otimes N S_{S}$, where $N S_{S}$ is the Artin motive corresponding to the Néron-Severi lattice of $S$. Hence $\operatorname{det}\left(h^{2}(S)\right)=\mathbb{L}^{\rho} \otimes \operatorname{det}\left(N S_{S}\right)$, where $\rho$ is the Picard number. For examples where the action of the absolute Galois group of $k$ on $N S_{S}$ has nontrivial determinant $d \in k^{*} / k^{* 2}$, we may take for $S$ the blow-up of $\mathbb{P}^{2}$ at $\{(0: \sqrt{d}),(0:-\sqrt{d})\}$ (I thank Colliot-Thélène for pointing this out): this shows that all Artin motives of square $\mathbf{1}$ are caught thusly. This also incidentally explains the sign $(-1)^{\mu}$ in $[43$, p. 9]. I expect that this is typical of how nontrivial Artin motives may occur.

d) The class of varieties $X$ such that $h(X) \in K_{0}^{\prime}(\mathcal{M}(k))$ is closed under products; it is also closed under projective bundles and blow-ups with smooth centres, in the sense that of $[h(X)] \in K_{0}^{\prime}(\mathcal{M}(k))$ and $Z$ is a closed smooth subvariety of $X$ such that $[h(Z)] \in K_{0}^{\prime}(\mathcal{M}(k))$, then $\left[h\left(B l_{Z}(X)\right)\right] \in K_{0}^{\prime}(\mathcal{M}(k))$. Thus surfaces $S$ such that $h(S)$ verifies (14.1) include rational surfaces, ruled surfaces, products of two curves and abelian surfaces. The first open case for (14.1) seems to be that of the motive of a K3 surface. 


\section{An EXtension: Zeta FUnCTIONS OF ENDOMORPHismS}

15.1. Definition. Let $\mathcal{M}$ be as in $\S 11$. The category $\mathcal{E}$ nd $(\mathcal{M})$ of endomorphisms of $\mathcal{M}$ has objects the pairs $(M, f)$ where $M \in \mathcal{M}$ and $f \in \operatorname{End}_{\mathcal{M}}(M)$; a morphism from $(M, f)$ to $(N, g)$ is an element $\varphi \in \mathcal{M}(M, N)$ such that $\varphi f=g \varphi$. This is a tensor $K$-category for the rule $(M, f) \otimes(N, g)=(M \otimes N, f \otimes g)$ and the various constraints induced by those of $\mathcal{M}$.

The following lemma is easily proven using [52, Ch. I, (3.2.3.5) and (3.2.3.6)]:

15.2. Lemma. An object $(M, f) \in \mathcal{E} \operatorname{nd}(\mathcal{M})$ is dualisable if and only if $M$ is dualisable and $f$ is invertible; then $(M, f)^{*}=\left(M^{*}, f^{*}\right)$ where $f^{*}={ }^{t} f^{-1}$.

15.3. Definition. We denote by $\mathcal{A u t}(\mathcal{M})$ the full subcategory of $\mathcal{E} \operatorname{nd}(\mathcal{M})$ consisting of dualisable objects.

15.4. Lemma. $(M, f) \in \mathcal{E} \operatorname{nd}(\mathcal{M})$ is finite dimensional if and only if $M \in \mathcal{M}$ is finite dimensional.

Proof. "Only if" is obvious. For "if", let $\varphi: M^{+} \oplus M^{-} \stackrel{\sim}{\longrightarrow} M$ be a decomposition of $M$ into a sum of a positive and a negative object. Modulo $\mathcal{N}$, we may write $\bar{f}=\bar{\varphi}\left(\bar{f}^{+} \oplus \bar{f}^{-}\right) \bar{\varphi}^{-1}$ : in other words, $\bar{\varphi}$ defines an isomorphism $\left(\bar{M}^{+}, \bar{f}^{+}\right) \oplus\left(\bar{M}^{-}, \bar{f}^{-}\right) \stackrel{\sim}{\longrightarrow}(\bar{M}, \bar{f})$ in $\mathcal{E} \operatorname{nd}(\mathcal{M} / \mathcal{N})=\mathcal{E} \operatorname{nd}(\mathcal{M}) / \mathcal{N}$. Since $\mathcal{N}(M, M)$ is nilpotent, so is its subgroup $\mathcal{N}((M, f),(M, f))$; thus this isomorphism lifts as a decomposition $\varphi^{\prime}:(M, f) \stackrel{\sim}{\longrightarrow}\left(M^{+}, f^{+}\right) \oplus\left(M^{-}, f^{-}\right)$for suitable $\varphi^{\prime}, f^{+}, f^{-}$.

The definitions and results on motivic zeta functions specialise to the cases of $\mathcal{E} \operatorname{nd}(\mathcal{M})$ and $\mathcal{A u t}(\mathcal{M})$. We have a ring homomorphism

$$
\begin{aligned}
K_{0}(\mathcal{E} \operatorname{nd}(\mathcal{M})) & \stackrel{\operatorname{tr}}{\longrightarrow} K \\
{[M, f] } & \mapsto \operatorname{tr}_{\mathcal{M}}(f)
\end{aligned}
$$

where $\operatorname{tr}_{\mathcal{M}}$ is the trace in $\mathcal{M}$.

15.5. Proposition. For any $(M, f) \in \mathcal{E} \operatorname{nd}(\mathcal{M})$, the motivic zeta function $Z_{\mathcal{M}}(M, f, T) \in$ $K_{0}(\mathcal{E} \operatorname{nd}(\mathcal{M}))[[T]]$ specialises via $\operatorname{tr}$ to the ordinary zeta function of $f$ (see $\left.\S 3\right)$ :

$$
Z(f ; T)=\exp \left(\sum_{n \geq 1} \operatorname{tr}\left(f^{n}\right) \frac{T^{n}}{n}\right) .
$$


In particular, $Z(f, T)$ is rational if $M$ is finite dimensional, and if $f$ is moreover invertible it satisfies the functional equation

$$
Z\left({ }^{t} f^{-1} ; T^{-1}\right)=\operatorname{det}(f)(-T)^{\chi(M)} Z(M, f ; T)
$$

where $\operatorname{det}(f)$ is defined in Proposition 11.3 and its proof.

Proof. By [4, Prop. 7.2.4], we have

$$
\operatorname{tr}_{\mathcal{M}} S^{n}(f)=\frac{1}{n !} \sum_{\sigma \in \mathfrak{S}_{n}} \prod_{k} \operatorname{tr}_{\mathcal{M}}\left(f^{l_{k}(\sigma)}\right)
$$

where $l_{1}(\sigma), \ldots, l_{p}(\sigma)$ are the lengths of the disjoint cycles constituting $\sigma$. The first formula then follows from the power series identity

$$
\exp \left(\sum_{n=1}^{\infty} x_{n} \frac{t^{n}}{n}\right)=\sum_{m=0}^{\infty} \frac{t^{m}}{m !} \sum_{\sigma \in \mathfrak{S}_{m}} \prod_{k=1}^{p} x_{l_{k}(\sigma)}
$$

This formula should be classical and goes presumably back to Euler [21]; here is a simple proof by Marc Hindry, following a hint of Georges Skandalis:

Let $k=\left(k_{1}, \ldots, k_{p}\right)$ be such that $k_{1}+2 k_{2}+\cdots+p k_{p}=m$. Let $H_{k}$ be the subset of $\mathfrak{S}_{m}$ made of those permutations decomposing into $k_{1}$ cycles of length $1, k_{2}$ cycles of length 2 , etc. This is a conjugacy class and the centraliser of such a permutation has cardinal $k_{1} ! \ldots k_{p} ! 2^{k_{2}} \ldots p^{k_{p}}$, so that

$$
\left|H_{k}\right|=\frac{m !}{k_{1} ! \ldots k_{p} ! 2^{k_{2}} \ldots p^{k_{p}}} .
$$


Thus

$$
\begin{aligned}
\exp \left(\sum_{n=1}^{\infty} x_{n} \frac{t^{n}}{n}\right) & =\prod_{n=1}^{\infty} \exp \left(x_{n} \frac{t^{n}}{n}\right) \\
& =\prod_{n=1}^{\infty} \sum_{k_{n}=0}^{\infty}\left(x_{n} \frac{t^{n}}{n}\right)^{k_{n}} \frac{1}{k_{n} !} \\
& =\sum_{k_{1}, \ldots, k_{N}} \frac{x_{1}^{k_{1}} \ldots x_{N}^{k_{N}}}{k_{1} ! \ldots k_{N} !} \prod_{n=1}^{N}\left(\frac{t^{n}}{n}\right)^{k_{n}} \\
& =\sum_{k_{1}, \ldots, k_{N}} \frac{x_{1}^{k_{1}} \ldots x_{N}^{k_{N}}}{k_{1} ! \ldots k_{N} !} \frac{t^{k_{1}+2 k_{2}+\cdots+N k_{N}}}{2^{k_{1}} \ldots N^{k_{N}}} \\
& =\sum_{m=0}^{\infty} t^{m} \sum_{k_{1}+2 k_{2}+\cdots+N k_{N}=m} \frac{x_{1}^{k_{1}} \ldots x_{N}^{k_{N}}}{k_{1} ! \ldots k_{N} ! 2^{k_{1}} \ldots N^{k_{N}}} \\
& =\sum_{m=0}^{\infty} \frac{t^{m}}{m !} \sum_{k_{1}+2 k_{2}+\cdots+N k_{N}=m} x_{1}^{k_{1}} \ldots x_{N}^{k_{N}}\left|H_{k}\right| \\
& =\sum_{m=0}^{\infty} \frac{t^{m}}{m !} \sum_{\sigma \in \mathfrak{S}_{m}} \prod_{k} x_{l_{k}(\sigma)} .
\end{aligned}
$$

Note that $\chi(M, f)=\chi(M)$ (e.g. use the $\otimes$-functor $(M, f) \mapsto M$ from $\mathcal{A u t}(\mathcal{M})$ to $\mathcal{M})$. In view of Theorem 12.1, to see the second formula, it therefore suffices to check $\operatorname{tr}[\operatorname{det}(M, f)]=(-1)^{\chi^{-}(M)} \operatorname{det}(f)$. But since $\operatorname{End}_{\mathcal{M}}(\operatorname{det}(M))=K$, we have

$$
\operatorname{tr}[\operatorname{det}(M, f)]=\chi(\operatorname{det}(M)) \operatorname{det}(f)=(-1)^{\chi^{-}(M)} \operatorname{det}(f) .
$$

15.6. Remarks. 1) For $n \geq 1$, let $\Psi^{n}$ be the $n$-th Adams operation. A suggestive form of the identity in Proposition 15.5 is

$$
\operatorname{tr} \Psi^{n}([M, f])=\operatorname{tr}_{\mathcal{M}}\left(f^{n}\right) ; \quad \operatorname{tr}[\underline{\operatorname{det}}(M, f)]=(-1)^{\chi(M)} \operatorname{det}(f)
$$

(compare Formula (1) in $[6, \S 5])$.

2) This gives another proof of Theorem 3.2 in the special case of finite dimensional objects (recall that, with the terminology of Part I, a finite dimensional object is of integral type by Proposition $2.2 \mathrm{e})$ ). In particular, the $\operatorname{det}(f)$ appearing in that theorem and in Proposition 15.5 coincide. 
3) Let $F$ be a $\otimes$-endomorphism (hence isomorphism) of the identity functor of $\mathcal{M}$. This defines a tensor functor

$$
\begin{aligned}
\mathcal{M} & \rightarrow \mathcal{A u t}(\mathcal{M}) \\
M & \left.\mapsto M, F_{M}\right) .
\end{aligned}
$$

In the case where $k=\mathbf{F}_{q}$, this is a way to explain how one recovers the classical zeta function from the motivic one. (See also Part IV.)

4) For $(M, f),(N, g) \in \mathcal{E} \operatorname{nd}(\mathcal{M})$, let

$$
\mathcal{I}((M, f),(N, g))=\{\varphi: M \rightarrow N \mid g \varphi=\varphi f=0\} .
$$

This defines a $\otimes$-ideal; note that $\mathcal{E} \operatorname{nd}(\mathcal{M}) / \mathcal{I}$ is used to construct the pseudoabelian envelope of $\mathcal{M}$. The homomorphism tr factors through $K_{0}(\mathcal{E} \operatorname{nd}(\mathcal{M}) / \mathcal{I})$.

\section{Part IV. An abstract version of the Tate(-Beilinson) conjecture}

\section{INTRODUCTION}

In this part, I formulate a version of the Tate conjecture for motives over a finite field in an abstract set-up. My initial motivation was to see what the multiplicities of Part I had to say on this conjecture; they are actually essential in the proof of Proposition 17.6. It turns out that most of the known equivalent versions of the Tate conjecture carry out in this abstract context: see Theorem 17.2 and Corollary 18.2. The proof of [32] that under the Tate conjecture and a nilpotence hypothesis, rational and numerical equivalences agree over a finite field also carries out abstractly: see Theorem 19.1.

Terminology and notation are the same as in Part I.

\section{Automorphisms of the identity FUnCtor}

Let $\mathcal{A}$ be a rigid $K$-category, and let $F$ be an $\otimes$-endomorphism of the identity functor of $\mathcal{A}$. By [52, I.5.2.2], $F$ is then an isomorphism. Concretely, $F$ is given by an automorphism $F_{M} \in \operatorname{End}(M)$ for every object $M \in \mathcal{A} ; F_{M}$ is natural in 
$M$ (in particular, it is central in $\operatorname{End}(M)$ ), and further:

$$
\begin{aligned}
F_{M \oplus N} & =F_{M} \oplus F_{N} \\
F_{M \otimes N} & =F_{M} \otimes F_{N} \\
F_{M^{*}} & ={ }^{t} F_{M}^{-1}(c f .[52, \mathrm{I},(3.2 .3 .6)]) .
\end{aligned}
$$

16.1. Definition. The zeta function (relative to $F$ ) of an object $M \in \mathcal{A}$ is

$$
Z_{F}(M, t)=Z\left(F_{M}, t\right)
$$

16.2. Lemma. The zeta function is additive in $M$ :

$$
Z_{F}(M \oplus N, t)=Z_{F}(M, t) Z_{F}(N, t) .
$$

It is multiplicative in $M$ in the following sense:

$$
Z_{F}(M \otimes N, t)=Z_{F}(M, t) * Z_{F}(N, t)
$$

where $*$ is the unique law on $1+t \bar{K}[[t]]$ such that, identically, $f *(g h)=(f * g)(f * h)$ and

$$
(1-a t)^{-1} *(1-b t)^{-1}=(1-a b t)^{-1} .
$$

(Explicitly: if $f(t)=\exp \left(\sum_{n \geq 1} a_{n} \frac{t^{n}}{n}\right)$ and $g(t)=\exp \left(\sum_{n \geq 1} b_{n} \frac{t^{n}}{n}\right)$, then $(f *$ $g)(t)=\exp \left(\sum_{n \geq 1} a_{n} b_{n} \frac{t^{n}}{n}\right)$.)

If moreover $\mathcal{A}$ is semi-simple of integral type, then

(1) $Z_{F}(M, t) \in K(t)$ for any $M \in \mathcal{A}$;

(2) $Z_{F}\left(M^{*}, t^{-1}\right)=(-t)^{\chi(M)} \operatorname{det}\left(F_{M}\right) Z_{F}(M, t)$;

(3) for $S$ simple,

$$
Z_{F}(S, t)=P_{S}(t)^{-\chi(S) / \operatorname{deg}\left(F_{S}\right)}
$$

where $P_{S}(t)$ is the inverse minimum polynomial of $F_{S}$ over $K$ and $\operatorname{deg}\left(F_{S}\right)=$ $\operatorname{deg}\left(P_{S}\right)=\left[K\left[F_{S}\right]: K\right]$.

Proof. Additivity is obvious; multiplicativity follows from the identities

$$
\operatorname{tr}\left(F_{M \otimes N}^{n}\right)=\operatorname{tr}\left(F_{M}^{n} \otimes F_{N}^{n}\right)=\operatorname{tr}\left(F_{M}^{n}\right) \operatorname{tr}\left(F_{N}^{n}\right) .
$$

(1), (2) and (3) follow from Theorem 3.2: (1) from part a), (2) from part b) by noting that $Z\left({ }^{t} F_{S}^{-1}, t^{-1}\right)=Z\left(F_{S}^{-1}, t^{-1}\right)$, and (3) from part a) again by noting that $F_{S}$ is in the centre of $\operatorname{End}_{\mathcal{A}}(S)$ (use Proposition 2.2 a)). 


\section{The SEMI-SIMPle CASE}

17.1. Definition. In the above, suppose $\mathcal{A}$ semi-simple of integral type. We say that $(\mathcal{A}, F)$ verifies the Tate conjecture if, for any $M \in \mathcal{A}, K\left[F_{M}\right]$ is the centre of $\operatorname{End}_{\mathcal{A}}(M)$.

17.2. Theorem (cf. [24, Th. 2.7]). Let $\mathcal{A}$ be a semi-simple rigid pseudo-abelian $K$-category of integral type (see Definition 2.1), and let $F \in \operatorname{Aut}^{\otimes}\left(I d_{\mathcal{A}}\right)$. Then the following conditions are equivalent:

(i) Given a simple object $S \in \mathcal{A}, F_{S}=1_{S}$ implies $S=\mathbf{1}$.

(ii) For any $M \in \mathcal{A}, \operatorname{ord}_{t=1} Z_{F}(M, t)=-\operatorname{dim}_{K} \mathcal{A}(\mathbf{1}, M)$.

(iii) For $S, T \in \mathcal{A}$ simple, $P_{S}=P_{T} \Rightarrow S \simeq T$ (see Lemma 16.2 (3)).

(iv) For $M, N \in \mathcal{A}, Z_{F}(M, t)=Z_{F}(N, t) \Rightarrow M \simeq N$.

(v) $(\mathcal{A}, F)$ verifies the Tate conjecture.

Moreover, these conditions imply (cf. Theorem 6.3 d)):

(vi) For any simple $S,|\mu(S)|=1$ and $K\left[F_{S}\right]$ is the centre of the algebra $\operatorname{End}_{\mathcal{A}}(S)$.

Proof. We shall prove the following implications:

(i) $\Rightarrow$ (ii) $\Rightarrow$ (iii) $\Rightarrow$ (iv) $\Rightarrow$ (i)

(ii) $\Rightarrow($ vi)

(iii) + (vi) $\Rightarrow$ (v) $\Rightarrow$ (iii).

(i) $\Rightarrow$ (ii): both sides are additive in $M$ so we may assume $M$ simple. If $M=\mathbf{1}$, $Z_{F}(M, t)=1 /(1-t)$ and the formula is true. If $M \neq 1$, Lemma $16.2(3)$ and the hypothesis show that $\operatorname{ord}_{t=1} Z_{F}(M, t)=0$ and the formula is also true.

(ii) $\Rightarrow$ (iii): Consider $f(t)=Z_{F}\left(S^{*} \otimes T, t\right)$. By Lemma 16.2, Formulas (2), (3) and the multiplicativity rule, we see that

$$
f(t)=\prod_{i, j}\left(1-\alpha_{i} \alpha_{j}^{-1} t\right)^{m}
$$

where $m=-\frac{\chi(S)}{\operatorname{deg}\left(F_{S}\right)} \frac{\chi(T)}{\operatorname{deg}\left(F_{T}\right)}$ and the $\alpha_{i}$ are the roots of the irreducible polynomial $P_{S}=P_{T}$ in a suitable extension of $K$. Note that $\chi(S) \chi(T) \neq 0$ : this follows from Propositions 1.2 and $2.2 \mathrm{a}$ ). The above formula then shows that $\operatorname{ord}_{t=1} Z_{F}(M, t) \neq$ 
0 . Hence (by (ii)) $0 \neq \mathcal{A}\left(\mathbf{1}, S^{*} \otimes T\right) \simeq \mathcal{A}(S, T)$, and $S \simeq T$ by Schur's lemma (recall that $\mathcal{A}$ is abelian, cf. Remark 1.4).

(iii) $\Rightarrow$ (iv): write $M=\bigoplus_{i \in I} S_{i}^{m_{i}}$ and $N=\bigoplus_{i \in I} S_{i}^{n_{i}}$, where $S_{i}$ runs through a set of representatives of the isomorphism classes of simple objects of $\mathcal{A}$. We then have, by Lemma $16.2(3)$ :

$$
\begin{aligned}
& Z_{F}(M, t)=\prod_{i \in I} P_{S_{i}}(t)^{-m_{i} \chi\left(S_{i}\right) / \operatorname{deg}\left(F_{S_{i}}\right)} \\
& Z_{F}(N, t)=\prod_{i \in I} P_{S_{i}}(t)^{-n_{i} \chi\left(S_{i}\right) / \operatorname{deg}\left(F_{S_{i}}\right)} .
\end{aligned}
$$

By hypothesis, the $P_{S_{i}}(t)$ are pairwise distinct irreducible polynomials with constant term 1 ; then $Z_{F}(M, t)=Z_{F}(N, t)$ implies $m_{i}=n_{i}$ for all $i$, hence $M \simeq N$.

(iv) $\Rightarrow\left(\right.$ i): by hypothesis and Lemma $16.2(3), Z_{F}(S, t)=(1-t)^{-\chi(S)}$. Thus $Z_{F}(S, t)=Z_{F}(\mathbf{1}, t)^{\chi(S)}$. If $\chi(S)<0$, this gives $S^{-\chi(S)} \simeq \mathbf{1}$, which implies $\chi(S)=-1$ and $S \simeq \mathbf{1}$, which is absurd since $\chi(\mathbf{1})=1$. Thus $\chi(S) \geq 0$, hence $S \simeq \mathbf{1}^{\chi(S)}$, hence $S \simeq \mathbf{1}$ since $S$ is simple.

(ii) $\Rightarrow(v i)$ : the same computation as in the proof of (ii) $\Rightarrow$ (iii) gives

$$
\begin{aligned}
\delta(S) d(S)^{2}=\operatorname{dim} \operatorname{End}_{\mathcal{A}}(S) & =-\operatorname{ord}_{t=1} Z\left(S^{*} \otimes S, t\right) \\
& =\left(\frac{\chi(S)}{\operatorname{deg}\left(F_{S}\right)}\right)^{2} \operatorname{ord}_{t=1} \prod_{i, j}\left(1-\alpha_{i} \alpha_{j}^{-1} t\right)=\frac{\chi(S)^{2}}{\operatorname{deg}\left(F_{S}\right)}
\end{aligned}
$$

Using the identity $\chi(S)=\mu(S) d(S) \delta(S)$ (cf. Proposition 2.2 a)), we get

$$
\operatorname{deg}\left(F_{S}\right)=\delta(S) \mu(S)^{2} .
$$

But $\operatorname{deg}\left(F_{S}\right) \mid \delta(S)$, hence $\delta(S)=\operatorname{deg}\left(F_{S}\right)$ and $\mu(S)^{2}=1$.

(iii) $+(\mathrm{vi}) \Rightarrow(\mathrm{v})$ : Let $M=\bigoplus_{i} S_{i}^{m_{i}}$ with $m_{i}>0$ and the $S_{i}$ simple and pairwise nonisomorphic. Then

$$
\operatorname{End}_{\mathcal{A}}(M)=\prod_{i} M_{m_{i}}\left(\operatorname{End}_{\mathcal{A}}\left(S_{i}\right)\right)
$$

hence the centre of $\operatorname{End}_{\mathcal{A}}(M)$ is the product of the centres of the $\operatorname{End}_{\mathcal{A}}\left(S_{i}\right)$. By (vi), each of these centres is generated by $F_{S_{i}}$; by (iii), the $P_{S_{i}}$ are pairwise 
distinct irreducible polynomials, hence the inverse minimum polynomial of $F_{M}$ must be divisible by their product.

(v) $\Rightarrow$ (iii) (compare [24]): if $P_{S}=P_{T}$ but $S \not T T$, then $\operatorname{End}_{\mathcal{A}}(S \oplus T)=$ $\operatorname{End}_{\mathcal{A}}(S) \times \operatorname{End}_{\mathcal{A}}(T)$, with centre containing $L \times L$ for $L=K\left[F_{S}\right]=K\left[F_{T}\right]$. But $F_{S \oplus T}$ is killed by $P_{S}=P_{T}$, a contradiction.

17.3. Remark. Condition (vi) is really weaker than the others: take $F=1$ in $\mathcal{A}$ the category of linear representations of a nontrivial finite abelian group over $K$ algebraically closed.

17.4. Proposition. Let $\mathcal{A}$ be semi-simple of integral type and let $F \in \operatorname{Aut}^{\otimes}\left(\operatorname{Id}_{\mathcal{A}}\right)$. a) The Tate conjecture is true for $(\mathcal{A}, F)$ if and only if it is true for $\left(\mathcal{A}^{\natural}, F\right)$, where $\mathcal{A}^{\natural}$ is the pseudo-abelian envelope of $\mathcal{A}$ and $F$ is extended to $\mathcal{A}^{\natural}$ naturally.

b) If $\mathcal{A}$ is geometrically of integral type, the Tate conjecture is invariant under extension of scalars: if $L$ is an extension of $K$, then $(\mathcal{A}, F)$ verifies the Tate conjecture if and only if $\left(\mathcal{A}_{L}, F\right)$ verifies the Tate conjecture.

Proof. a) "If" is obvious. For "only if", let $M=(N, e) \in \mathcal{A}^{\natural}$ where $N \in \mathcal{A}$ and $e$ is an idempotent of $N$. Write $M=\bigoplus_{i \in I} S_{i}^{m_{i}}$ and $N=\bigoplus_{i \in I} S_{i}^{n_{i}}$ as in the proof of Theorem 17.2, (iii) $\Rightarrow$ (iv). We have

$$
\operatorname{End}(M)=\prod_{i} M_{m_{i}}\left(\operatorname{End}\left(S_{i}\right)\right), \quad \operatorname{End}(N)=\prod_{i} M_{n_{i}}\left(\operatorname{End}\left(S_{i}\right)\right) .
$$

Letting $Z_{i}$ denote the centre of $\operatorname{End}\left(S_{i}\right)$, we get

$$
Z(\operatorname{End}(M))=\prod_{m_{i}>0} Z_{i}, \quad Z(\operatorname{End}(N))=\prod_{n_{i}>0} Z_{i}
$$

By hypothesis, $Z(\operatorname{End}(N))$ is generated by $F_{N}$ as a $K$-algebra; this implies that $Z_{i}$ is generated by $F_{S_{i}}$ for all $i$ and that the $P_{S_{i}}$ are pairwise distinct. Hence $F_{M}$ generates $Z(\operatorname{End}(M))$ as well.

b) This is obvious since the centre of a semi-simple algebra commutes with extension of scalars in characteristic 0 .

17.5. Corollary. If $(\mathcal{A}, F)$ verifies the Tate conjecture, then the conditions of Theorem 17.2 hold in $\mathcal{A}$ even if $\mathcal{A}$ is not pseudo-abelian. 
Proof. This is obvious (by passing to $\mathcal{A}^{\natural}$ ) except for (ii) and (iv); but by Proposition $17.4 \mathrm{a}),\left(\mathcal{A}^{\natural}, F\right)$ verifies the Tate conjecture; by Theorem $17.2, \mathcal{A}^{\natural}$ also verifies conditions (ii) and (iv), which a fortiori hold in its full subcategory $\mathcal{A}$.

17.6. Proposition. Suppose that $\mathcal{A}$ is pseudo-abelian and that $(\mathcal{A}, F)$ verifies the Tate conjecture. Then

a) $\mathcal{A}$ is Kimura-O'Sullivan (any $M \in \mathcal{A}$ is finite-dimensional). More precisely, there exists a unique $\otimes-\mathbf{Z} / 2$-grading of $\mathcal{A}$ such that $S$ simple is positive (resp. negative) if and only if $\chi(S)>0$ (resp. $<0)$.

b) $\mathcal{A}$ is super-tannakian (tannakian if we change the commutativity constraint as in $[4$, Th. 9.2.1 b)]).

Proof. a) By Lemma 4.3 and Proposition 17.4, we may assume $K$ algebraically closed. In view of Condition (vi) of Theorem 17.2 and Proposition 4.5, it suffices to prove that the tensor product of two simple objects $S, S^{\prime}$ is of constant sign in the sense of Definition 4.1.

Since $K$ is algebraically closed, $\operatorname{End}(S)=\operatorname{End}\left(S^{\prime}\right)=K$ and $F_{S}, F_{S^{\prime}}$ are scalars $\lambda, \lambda^{\prime}$. Hence $F_{S \otimes S^{\prime}}=F_{S} \otimes F_{S^{\prime}}$ is the scalar $\lambda \lambda^{\prime}$. Let $T$ be a simple summand of $S \otimes S^{\prime}$, corresponding to the idempotent $e$ of $\operatorname{End}\left(S \otimes S^{\prime}\right)$. Then $F_{T}=e F_{S \otimes S^{\prime}}=$ $\lambda \lambda^{\prime} e$. Hence $P_{T}=1-\lambda \lambda^{\prime} t$ is independent of $T$, which implies by Condition (iii) of Theorem 17.2 that $S \otimes S^{\prime}$ is isotypical; in particular, it is of constant sign.

"More precisely" follows from [4, th. 9.2.1 a)].

b) Since every object of $\mathcal{A}$ is finite-dimensional, it is Schur-finite [13, 1.13]): the first assertion then follows from the main theorem of ibid. The second one follows from [4, Th. 9.2.1 c)] (or [12, th. 7.1]).

17.7. Example. Suppose $K$ algebraically closed: then every simple object $S$ of $\mathcal{A}$ is invertible (see Condition (iv) in Proposition 4.5). The endomorphism $F_{S}$ is a scalar $\lambda_{S} \in K^{*}$, and $S$ is characterised by $\lambda_{S}$. The set of $\lambda_{S}$ forms a subgroup $\Lambda$ of the multiplicative group $K^{*}$, and is provided with a canonical homomorphism $\Lambda \rightarrow \mathbf{Z} / 2$ sending $\lambda_{S}$ to the sign of $S$. There exists a unique super-fibre functor $\omega: \mathcal{A} \rightarrow \operatorname{Vec}_{K}^{ \pm}$(realisation in the sense of Definition 5.1), characterised by $\omega(S)=K^{\varepsilon(S)}$, where $\varepsilon(S)$ is the sign of $S$ (Definition 4.1). The corresponding "super-tannakian group" is the affine group scheme of multiplicative type $T$ with character group $\Lambda$, provided with the canonical homomorphism $\mu_{2} \rightarrow T$ dual to the sign homomorphism. This makes it easy to compute the object in the 
ind-category $\operatorname{ind}(\mathcal{A})$ representing the internal (super-)fundamental group $\mathcal{G}$ of $\mathcal{A}$ in the sense of $[12, \S 8]$ : it is given by $M^{*} \otimes M$, where $M$ is the direct sum of a set of representatives of simple objects, with multiplicity 1 . It follows that $\mathcal{G}$ is represented by the same object if $K$ is no longer algebraically closed. The automorphism $F$ defines a canonical "rational point" of $\mathcal{G}$, which generates a dense "subgroup".

\section{The homological CASE}

Let $\mathcal{A}$ be of homological type, provided with a faithful realisation functor $H: \mathcal{A} \rightarrow \operatorname{Vec}_{L}^{ \pm}$. Let $F \in \operatorname{Aut}^{\otimes}\left(I d_{\mathcal{A}}\right)$, and let us still denote by $F$ its image in $\operatorname{Aut}^{\otimes}\left(I d_{\overline{\mathcal{A}}}\right)$, where $\overline{\mathcal{A}}=\mathcal{A} / \mathcal{N}(\mathcal{N}$ is as in $(1.2))$. Note that $F$ acts on $H$ by functoriality.

18.1. Theorem. Consider the following conditions on an object $M \in \mathcal{A}$ :

(i) $\bar{M} \in \overline{\mathcal{A}}$ verifies Condition (ii) of Theorem 17.2.

(ii) The map $\mathcal{A}(\mathbf{1}, M) \otimes_{K} L \rightarrow H(M)^{F}$ is surjective and the composition $H(M)^{F} \rightarrow H(M) \rightarrow H(M)_{F}$ is an isomorphism (semi-simplicity at 1 ).

(iii) The map $\mathcal{A}(\mathbf{1}, M) \otimes_{K} L \rightarrow H(M)^{F}$ is surjective and $\mathcal{N}(\mathbf{1}, M)=0$.

(iv) The sign property (Definition 5.8) holds for $M$.

(v) $H^{-}(M)^{F}=0$.

(vi) The polynomials $\operatorname{det}\left(1-F_{M} t \mid H^{+}(M)\right)$ and $\operatorname{det}\left(1-F_{M} t \mid H^{-}(M)\right)$ are coprime.

Then

(1) (i) + (v) $\Longleftrightarrow$ (ii) + (iii).

(2) (i) + (iv) $\Rightarrow$ (v).

(3) (vi) $\Rightarrow$ (iv) and (vi) + (i) $\Rightarrow$ (v).

(4) (ii) for $M$ and $M^{*} \Longleftrightarrow$ (iii) for $M$ and $M^{*}$.

Proof. These are classical arguments that only need to be put straight in this abstract context.

Note that $H^{-}(\mathbf{1})=0$, so that $\mathcal{A}(\mathbf{1}, M) \otimes_{K} L \rightarrow H(M)^{F}$ actually lands into $H^{+}(M)^{F}$; denote its image by $\mathcal{A}(\mathbf{1}, M) L$. By definition of $\mathcal{N}$ and [5, Lemma 1], the projection $\mathcal{A}(\mathbf{1}, M) \otimes_{K} L \rightarrow \overline{\mathcal{A}}(\mathbf{1}, M) \otimes_{K} L$ factors through $\mathcal{A}(\mathbf{1}, M) L$. The 
diagram

gives the inequalities

$$
\begin{aligned}
\mathcal{A}(\mathbf{1}, M) L & \hookrightarrow H^{+}(M)^{F} \\
\text { surj } & \\
\overline{\mathcal{A}}(\mathbf{1}, \bar{M}) \otimes_{K} L &
\end{aligned}
$$

$$
\operatorname{dim}_{L} H^{+}(M)^{F} \geq \operatorname{dim}_{L} \mathcal{A}(\mathbf{1}, M) L \geq \operatorname{dim}_{K} \overline{\mathcal{A}}(\mathbf{1}, \bar{M}) .
$$

On the other hand,

$$
\begin{aligned}
& \operatorname{ord}_{t=1} Z_{F}(M, t)= \\
& \qquad \begin{aligned}
\operatorname{ord}_{t=1} \operatorname{det}\left(1-F_{M} t \mid H^{-}(M)\right) & -\operatorname{ord}_{t=1} \operatorname{det}\left(1-F_{M} t \mid H^{+}(M)\right) \\
& =\operatorname{dim}_{L} H^{-}(M)^{F^{\infty}}-\operatorname{dim}_{L} H^{+}(M)^{F^{\infty}}
\end{aligned}
\end{aligned}
$$

where $H^{ \pm}(M)^{F^{\infty}}$ denotes the characteristic subspace of $H^{ \pm}(M)$ for the eigenvalue 1 under the action of $F$.

(1) Suppose that $H^{-}(M)^{F}=0$. Then $H^{-}(M)^{F^{\infty}}=0$ and, under (i), we have

$\operatorname{dim}_{L} H^{+}(M)^{F} \geq \operatorname{dim}_{L} \mathcal{A}(\mathbf{1}, M) L \geq \operatorname{dim}_{K} \overline{\mathcal{A}}(\mathbf{1}, \bar{M})$

$$
=\operatorname{dim}_{L} H^{+}(M)^{F^{\infty}} \geq \operatorname{dim}_{L} H^{+}(M)^{F}
$$

hence we have equality everywhere, and (ii) and (iii) are true. Conversely, (ii) + (iii) gives isomorphisms $\overline{\mathcal{A}}(\mathbf{1}, M)_{L} \stackrel{\sim}{\longrightarrow} H(M)^{F} \stackrel{\sim}{\longrightarrow} H(M)^{F^{\infty}}$. In particular, $H^{-}(M)^{F}=0$ and we have $\operatorname{dim}_{K} \overline{\mathcal{A}}(\mathbf{1}, M)=\operatorname{dim}_{L} H^{+}(M)^{F^{\infty}}$, hence (i) and (v). Thus, (i) + (v) $\Longleftrightarrow$ (ii) + (iii).

(2) Under (iv), using Lemma 5.9 we may write $M=M^{+} \oplus M^{-}$, with $M^{+}$positive and $M^{-}$negative. To prove that $H^{-}(M)^{F}=0$, we may therefore consider separately the cases where $M$ is positive or negative.

If $M$ is positive, this is obvious. If $M$ is negative, we get, under (i):

$$
H^{+}(M)^{F}=\mathcal{A}(\mathbf{1}, M)=\overline{\mathcal{A}}(\mathbf{1}, \bar{M})=0
$$

since $\mathcal{A}(\mathbf{1}, M) \hookrightarrow H^{+}(M)^{F}$, and

$$
-\operatorname{dim} H^{-}(M)^{F^{\infty}}=\operatorname{dim} \overline{\mathcal{A}}(\mathbf{1}, \bar{M})=0
$$

which shows that (i) + (iv) $\Rightarrow(\mathrm{v})$. 
(3) For (vi) $\Rightarrow$ (iv), we reason as in [38, Proof of Th. 2]: there exists a polynomial $\Pi \in K[t]$ such that $\Pi$ is divisible by $P^{-}$and $\Pi-1$ is divisible by $P^{+}$, where $P^{\varepsilon}(t)=\operatorname{det}\left(t-F \mid H^{\varepsilon}(M)\right)$; then $\Pi(F) \in \operatorname{End}(M)$ is such that $H(\Pi(F))$ is the identity on $H^{+}(M)$ and is 0 on $H^{-}(M)$.

Suppose that (i) and (vi) hold and that $H^{-}(M)^{F} \neq 0$. Then $H^{+}(M)^{F}=$ $H^{+}(M)^{F^{\infty}}=0$. This implies that $\operatorname{ord}_{t=1} Z_{F}(M, t)>0$, which contradicts (i).

(4) The counit map $M \otimes M^{*} \rightarrow \mathbf{1}$ gives compatible pairings

$$
\begin{aligned}
\overline{\mathcal{A}}(\mathbf{1}, \bar{M}) \times \overline{\mathcal{A}}\left(\mathbf{1}, \bar{M}^{*}\right) & \rightarrow K \\
\mathcal{A}(\mathbf{1}, M) L \times \mathcal{A}\left(\mathbf{1}, M^{*}\right) L & \rightarrow L \\
H(M) \times H\left(M^{*}\right) & \rightarrow L .
\end{aligned}
$$

The first and last are perfect pairings: for the first, this follows from the definition of the ideal $\mathcal{N}$, and for the last, this follows from the structure of the tensor category $\operatorname{Vec}_{L}^{ \pm}$. Consider now the commutative diagram

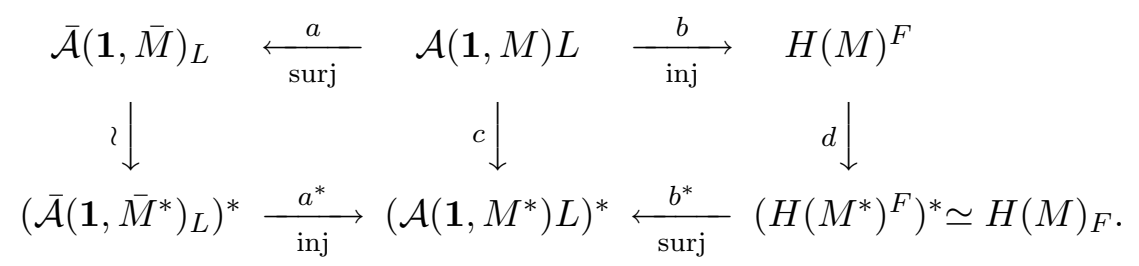

Notice that the right vertical map coincides with the one of (ii).

Now assume that $b$ and $b^{*}$ are isomorphisms. The diagram shows immediately that ( $a, a^{*}$ isomorphisms $) \Rightarrow$ ( $d$ isomorphism). Conversely, if $d$ is an isomorphism, so is $c$; but then, $a$ and $a^{*}$ must be isomorphisms. Finally, $a$ is an isomorphism $\Rightarrow \mathcal{A}(\mathbf{1}, M) \rightarrow \overline{\mathcal{A}}(\mathbf{1}, M) \otimes_{K} L$ is injective $\Rightarrow \mathcal{N}(\mathbf{1}, M)=0$, as desired.

18.2. Corollary (cf. $[57,2.9])$. Let $\mathcal{A}, H, F$ be as before, and suppose that $\mathcal{A}$ is pseudo-abelian. Consider the following conditions:

(i) The Tate conjecture holds for $(\overline{\mathcal{A}}, F)$.

(ii) $\mathcal{A} \rightarrow \overline{\mathcal{A}}$ is an equivalence of categories and $H$ induces a fully faithful functor

$$
\tilde{H}: \overline{\mathcal{A}}_{L} \rightarrow \operatorname{Rep}_{L}(F)_{s s}^{ \pm}
$$


where the right hand side denotes the $\otimes$-category of $\mathbf{Z} / 2$-graded finitedimensional $L$-vector spaces provided with the action of an automorphism $F$, this action being semi-simple.

(iii) Any $M \in \mathcal{A}$ has the sign property.

(iii') $\mathcal{A}$ is a Kimura-O'Sullivan category.

(iv) For any $M \in \mathcal{A}, H^{-}(M)^{F}=0$.

(v) For any $M \in \mathcal{A}$, the polynomials $\operatorname{det}\left(1-F_{M} t \mid H^{+}(M)\right)$ and $\operatorname{det}\left(1-F_{M} t \mid\right.$ $\left.H^{-}(M)\right)$ are coprime, and do not depend on the choice of the realisation $H$.

Then (i) $\Longleftrightarrow$ (ii) $\Rightarrow$ (v) $\Rightarrow$ (iii) $\Longleftrightarrow$ (iii') and (i) $\Longleftrightarrow$ (ii) $\Rightarrow$ (iv).

If all these conditions are verified, then for any simple object $S \in \mathcal{A}_{L}^{\natural}, \operatorname{End}(S)$ is commutative.

Proof. We shall prove the following implications:

(1) $(\mathrm{v}) \Rightarrow$ (iii);

(2) (ii) $\Rightarrow$ (i) + (iv);

(3) (i) $\Rightarrow$ (iii') $\Longleftrightarrow$ (iii);

(4) (i) + (iv) $\Rightarrow$ (ii);

(5) (i) + (iii) $\Rightarrow$ (iv);

(6) (i) + (ii) $+\left(\right.$ iii' $\left.^{\prime}\right) \Rightarrow(\mathrm{v})$.

(1) This is part of Point 3 of Theorem 18.1.

(2) If (ii) holds, then Conditions (ii) and (iii) of Theorem 18.1 hold for any $M$, hence so do its conditions (i) and (v) by Point 1 of this theorem. We conclude by Theorem 17.2.

(3) By Proposition 17.6, we have the implication (i) $\Rightarrow$ (iii'), and (iii') $\Longleftrightarrow$ (iii) by Lemma 5.9 , since $H$ is faithful.

(4) Suppose that (i) holds. Then Condition (ii) of Theorem 17.2 holds for any $\bar{M} \in \overline{\mathcal{A}}$. If moreover $H^{-}(M)^{F}=0$ for any $M \in \mathcal{A}$, Conditions (ii) and (iii) of Theorem 18.1 are verified for any $M \in \mathcal{A}$ by Point 1 of this theorem. Applying this to $M=P^{*} \otimes Q$ for some $P, Q \in \mathcal{A}$, the adjunction isomorphism

$$
\mathcal{A}(P, Q) \simeq \mathcal{A}\left(\mathbf{1}, P^{*} \otimes Q\right)
$$


shows that $\mathcal{N}(P, Q)=0$, hence a bijection

$$
\overline{\mathcal{A}}(P, Q) \otimes_{K} L \stackrel{\sim}{\longrightarrow} \operatorname{Hom}_{F}(H(P), H(Q)) .
$$

Moreover, since $\overline{\mathcal{A}}$ is semi-simple, $H\left(F_{M}\right)$ is a semi-simple endomorphism of $H(M)$ for any $M \in \mathcal{A}$.

(5) This is Point 2 of Theorem 18.1.

(6) Let $M \in \mathcal{A}$ : writing $M=M^{+} \oplus M^{-}$with $M^{+}$positive and $M^{-}$negative, we have $H^{+}(M)=H\left(M^{+}\right)$and $H^{-}(M)=H\left(M^{-}\right)$. Any simple direct summand of $M^{+}$(resp. of $M^{-}$) is positive (resp. negative), hence $M^{+}$and $M^{-}$have no common simple summand. By Condition (iii) of Theorem 17.2, $P_{M^{+}}$and $P_{M^{-}}$ are coprime, hence so are the polynomials $Z\left(M^{\varepsilon}, t\right)^{-1}=\operatorname{det}\left(1-F_{M^{\varepsilon}} \mid H\left(M^{\varepsilon}\right)\right)$, by Lemma 16.2 (see Formula (3)). This lemma also shows that they do not depend on the choice of $H$.

It remains to justify the last claim: it follows from Proposition 5.10 a) and Condition (vi) of Theorem 17.2.

18.3. Remark. In the classical case of motives over a finite field, Katz-Messing deduce Condition (iii) of Corollary 18.2 from (v) (= Deligne's affirmation of the Weil conjecture) in [38]: the above argument (1) is an abstraction of this. Amusingly, the argument of (6) shows that the Tate conjecture implies part of the Weil conjecture. Here is a partial strengthening in the case of a $\mathbf{Z}$-graded realisation functor:

18.4. Proposition. Suppose that the functor $H$ is obtained from a $\otimes$-functor $H^{*}: \mathcal{A} \rightarrow \operatorname{Vec}_{L}^{*}$ to $\mathbf{Z}$-graded finite-dimensional L-vector spaces via the functor $\operatorname{Vec}_{L}^{*} \rightarrow \operatorname{Vec}_{L}^{ \pm}$induced by the homomorphism $\mathbf{Z} \rightarrow\{ \pm 1\}$. Then, if the conditions of Corollary 18.2 are satisfied, the following properties hold for any $M \in \mathcal{A}$ :

(i) The "Künneth projectors" defining the decomposition

$$
H^{*}(M)=\bigoplus_{i \in \mathbf{Z}} H^{i}(M)
$$

are in the image of $H$.

(ii) The polynomials $\operatorname{det}\left(1-F_{M} t \mid H^{i}(M)\right)$ are pairwise coprime (we don't claim that they are independent of the choice of $\left.H^{*}\right)$. 
Proof. (i) By Condition (ii) of Corollary 18.2, the homomorphism

$$
H: \mathcal{A}(M, M) \rightarrow \operatorname{Rep}_{L}(F)^{*}\left(H^{*}(M), H^{*}(M)\right)
$$

is bijective. Since the "Künneth projectors" are central in the ring $\operatorname{Vec}_{L}^{*}\left(H^{*}(M), H^{*}(M)\right)$, they belong to $\operatorname{Rep}_{L}(F)^{*}\left(H^{*}(M), H^{*}(M)\right)$.

(ii) By (i), we may write $M=\bigoplus M_{i}$, with $H^{*}\left(M_{i}\right)=H^{i}(M)$. If $S_{i}, S_{j}$ are simple summands of $M_{i}$ and $M_{j}$ for $i \neq j$, then $S_{i}$ and $S_{j}$ are not isomorphic because $H^{*}\left(S_{i}\right)$ and $H^{*}\left(S_{j}\right)$ are not isomorphic. We conclude as in the proof of Corollary 18.2.

Suppose that $L=\bar{K}$ in Proposition 18.4. Then a Z-graded realisation $H^{*}$ corresponds to a Galois-invariant homomorphism $\varphi: \Lambda \rightarrow \mathbf{Z}$, where $\Lambda \subseteq \bar{K}^{*}$ is the group of eigenvalues of $F$ as in Example 17.7, lifting the sign homomorphism $\Lambda \rightarrow \mathbf{Z} / 2$. In general there can be many such homomorphisms: for example, one may take for $\mathcal{A}$ the full subcategory of $\operatorname{Rep}_{\mathbf{Q}}(\mathbf{Z})$ given by direct sums of 1-dimensional representations. Here $\Lambda=\mathbf{Q}^{*}$, and we may choose $\varphi=2 v_{p}$ for any $p$-adic valuation $v_{p}$ (the factor 2 is to ensure that there are no odd-dimensional $H^{*}$, since every object of $\mathcal{A}$ is positive here). In the classical case of numerical motives over a finite field $\mathbf{F}_{q}$, Deligne's proof of the Riemann hypothesis and Honda's theorem imply that $\Lambda$ is the set of all Weil $q$-numbers. We have a canonical homomorphism $\varphi: \Lambda \rightarrow \mathbf{Z}$ assigning to a Weil number its weight, and the kernel of $\varphi$ is divisible, which implies that $\varphi$ is unique up to scaling.

A fully satisfactory account of the situation in the abstract setting should include a treatment of the theory of weights along something like Grothendieck's standard conjectures: for this, one would have to consider polarisations and do positivity hypotheses. Since the present development is already exceedingly long, I leave this to the interested reader (see also Saavedra [52]).

\section{The Tate-Beilinson conjecture}

We conclude by transposing the argument of [32] to this abstract context.

19.1. Theorem (cf. [32, Th. 1]). Let $\mathcal{A}$ be a rigid $K$-category provided with a $\otimes-$ automorphism $F$ of the identity functor. Suppose that $\mathcal{N}$ is locally nilpotent (e.g. that $\mathcal{A}$ is a Kimura-O'Sullivan category), and that $\overline{\mathcal{A}}=\mathcal{A} / \mathcal{N}$ is semi-simple, of 
integral type and verifies the Tate conjecture relatively to $F$. Then $\mathcal{N}=0$, i.e. $\mathcal{A}=\overline{\mathcal{A}}$.

Proof. We note that the hypothesis on $\mathcal{N}$ implies that the functor $\mathcal{A} \rightarrow \overline{\mathcal{A}}$ is conservative. The argument is the same as in [32]: by rigidity it is sufficient to show that $\mathcal{A}(\mathbf{1}, M) \stackrel{\sim}{\longrightarrow} \overline{\mathcal{A}}(\mathbf{1}, \bar{M})$ for any $M \in \mathcal{A}$. By the nilpotence of $\mathcal{N}(M, M)$, we may lift to $\operatorname{End}_{\mathcal{A}}(M)$ an orthogonal system of idempotents of $\bar{M}$ corresponding to a decomposition in simple summands. This reduces us to $\bar{M}$ simple. There are two cases:

(1) $\bar{M} \simeq \mathbf{1}$. Then $M \simeq \mathbf{1}$ by conservativity, and both Hom groups are isomorphic to $K$.

(2) $\bar{M} \nsucceq \mathbf{1}$. Then $\overline{\mathcal{A}}(\mathbf{1}, \bar{M})=0$ and we have to show that $\mathcal{A}(\mathbf{1}, M)=0$. By Theorem $17.2(\mathrm{i}), F_{\bar{M}} \neq 1$, hence by conservativity, $1-F_{M}$ is an isomorphism. If now $f \in \mathcal{A}(\mathbf{1}, M)$, we have $f=F_{M} f$, hence $f=0$.

\section{REFERENCES}

[1] Y. André Pour une théorie inconditionnelle des motifs, Publ. Math. IHÉS 83 (1996), 5-49.

[2] Y. André Une introduction aux motifs: motifs purs, motifs mixtes, périodes, Panoramas et synthèses 17, SMF, 2004.

[3] Y. André, B. Kahn Construction inconditionnelle de groupes de Galois motiviques, C. R. Acad. Sci. Paris 334 (2002), 989-994.

[4] Y. André, B. Kahn Nilpotence, radicaux et structures monoïdales (with an appendix of P. O'Sullivan), Rend. Sem. Math. Univ. Padova 108 (2002), 107-291.

[5] Y. André and B. Kahn Erratum to Nilpotence, radicaux et structures monoïdales, Rend. Sem. Mat. Univ. Padova 113 (2005), 125-128.

[6] M. Atiyah, D. Tall Group representations, $\lambda$-rings and the J-homomorphism, Topology 8 (1969), 253-297.

[7] J. Ayoub The motivic vanishing cycles and the conservation conjecture, in Algebraic cycles and motives (in honour of J.P. Murre), Vol. 1, LMS Series 343, Cambridge University Press, 2007, 3-54.

[8] S. Bloch, A. Kas, D. Lieberman Zero cycles on surfaces with $p_{g}=0$, Compositio Math. 33 (1976), 135-145.

[9] A. Chambert-Loir Points rationnels et groupes fondamentaux : applications de la cohomologie p-adique (d'après P. Berthelot, T. Ekedahl, H. Esnault, etc.), Sém. Bourbaki, March 2003, Astérisque 294 (2004), 125-146.

[10] J.-L. Colliot-Thélène, J.-J. Sansuc La R-équivalence sur les tores, Ann. Sci. Éc. Norm. Sup. 10 (1977), 175-229. 
[11] P. Deligne La conjecture de Weil, II, Publ. Math. IHÉS 52 (1980), 137-252.

[12] P. Deligne Catégories tannakiennes, in The Grothendieck Festschrift (Vol. II), Progress in Math. 87, Birkhäuser, 1990, 111-196.

[13] P. Deligne Catégories tensorielles, Moscow Math. J. 2 (2002), 227-248.

[14] P. Deligne La catégorie des représentations du groupe symétrique $S_{t}$ lorsque $t$ n'est pas un entier naturel, Proc. Intern. Coll. on Alg. groups and Homog. Spaces, Jan. 2004, TIFR, Mumbai, 2004.

[15] A. del Padrone Schur-functors, nilpotency and motives, PhD Thesis, Univ. Genova, 2006.

[16] A. del Padrone Remarks on geometrically integral objects, preprint, 2007.

[17] A. Dold, D. Puppe Duality, trace and transfer, Proc. Int. Conf. in Geometric Topology (Warsaw, 1978), PWN, Warsaw, 1980, 81-102.

[18] T. Ekedahl Sur le groupe fondamental d'une variété unirationnelle, C. R. Acad. Sci. Paris 297 (1983), 627-629.

[19] H. Esnault Varieties over a finite field with trivial Chow group of zero-cycles have a rational point, Invent. Math. 151 (2003), 187-191.

[20] H. Esnault Deligne's integrality theorem in unequal characteristic and rational points over finite fields (with an appendix by Pierre Deligne and Esnault), Ann. of Math. 164 (2006), 715-730.

[21] L. Euler, Collected works.

[22] N. Fahkruddin, C.S. Rajan Congruences for rational points of varieties over finite fields, Math. Ann. 333 (2005), 797-809.

[23] W. Fulton Intersection theory, Springer, 1984.

[24] T. Geisser Tate's conjecture, algebraic cycles and rational K-theory in characteristic $p$, $K$-theory 13 (1998), 109-122.

[25] T. Graber, J. Harris, J. Starr Families of rationally connected varieties, J. Amer. Math. Soc. 16 (2003), 57-67.

[26] F. Heinloth A note on functional equations for zeta functions with values in Chow motives, Ann. Inst. Fourier 57 (2007), 1927-1945.

[27] A. Huber Slice filtration on motives and the Hodge conjecture (with an appendix by J. Ayoub), to appear in Math. Nachr.

[28] A.J. de Jong Smoothness, semi-stability and alterations, Publ. Math. IHÉS 83 (1996), 5193.

[29] U. Jannsen Motives, numerical equivalence and semi-simplicity, Invent. Math. 107 (1992), 447-452.

[30] U. Jannsen Motivic Sheaves and Filtrations on Chow groups, Proceedings of Symposia in Pure mathematics 55 (I) (1994), 245-302.

[31] B. Kahn Number of points of function fields over finite fields, first version of Part II, math.NT/0210202, Oct. 14, 2002.

[32] B. Kahn Équivalences rationnelle et numérique sur certaines variétés de type abélien sur un corps fini, Ann. Sci. Ec. Norm. Sup. 36 (2003), 977-2002.

[33] B. Kahn Motivic zeta functions of motives, first version of Part III, math. NT/0606424, June $16,2006$. 
[34] B. Kahn On the multiplicity of a motive, first version of Parts I and IV, math.AG/0610446, Feb. 5, 2007.

[35] B. Kahn, J. Murre, C. Pedrini On the transcendental part of the motive of a surface, in Algebraic cycles and motives (in honour of J.P. Murre), Vol. 2, LMS Series 344, Cambridge University Press, 2007, 143-202.

[36] B. Kahn, R. Sujatha Birational motives, I, preprint, 2002, http://www.math.uiuc.edu/K-theory \# 596.

[37] M. Kapranov The elliptic curve in the $S$-duality theory and Eisenstein series for Kac-Moody groups, math.AG/0001005, Feb. 21, 2000.

[38] N. Katz, W. Messing Some consequences of the Riemann hypothesis for varieties over finite fields, Invent. Math. 23 (1974), 73-77.

[39] S.-I. Kimura Chow motives are finite-dimensional, in some sense, Math. Ann. 331 (2005), 173-201.

[40] S.-I. Kimura A note on finite-dimensional motives, Algebraic cycles and motives (in honour of J.P. Murre), Vol. 2, LMS Series 344, Cambridge University Press, 2007, 203-213.

[41] S. Kleiman Algebraic cycles and the Weil conjectures, in Dix exposés sur la cohomologie des schémas, North-Holland, Amsterdam; Masson, Paris, 1968, 359-386.

[42] S. Kleiman Motives, Algebraic geometry, Oslo 1970, Proc. Fifth Nordic Summer School in Math., Wolters-Noordhoff, Groningen, 1972, 53-82.

[43] S. Kleiman The standard conjectures, in Motives, Proc. Symposia Pure Math. 55 (1), AMS, 1994, 3-20.

[44] F. Knudsen, D. Mumford The projectivity of the moduli space of stable curves. I. Preliminaries on "det" and "Div", Math. Scand. 39 (1976), 19-55.

[45] G. Lachaud, M. Perret, Un invariant birationnel des variétés de dimension 3 sur un corps fini, J. Algebraic Geom. 9 (2000), 451-458.

[46] J.S. Milne Motives over finite fields, Motives (Seattle, 1991), Proc. Sympos. Pure Math., 55, Part 1, Amer. Math. Soc., Providence, RI, 1994, 401-459.

[47] J.S. Milne The Tate conjecture for certain abelian varieties over finite fields, Acta Arith. 100 (2001), 135-166.

[48] J.S. Milne The Tate conjecture over finite fields, AIM talk, 2007, available on www . jmilne org.

[49] J.S. Milne, N. Ramachandran Motivic complexes over finite fields and the ring of correspondences at the generic point, preprint, 2006.

[50] C. Peters, J. Steenbrink Monodromy of variations of Hodge structures, Acta Appl. Math. 75 (2003), 183-194.

[51] C. Procesi Trace identities and standard diagrams, Ring Theory (Proc. Antwerp Conf. (NATO Adv. Study Inst., Univ. Antwerp, Antwerp, 1978), Lect. Notes in Pure and Appl. Math. 51, Dekker, 1979, 191-218.

[52] N. Saavedra Rivano Catégories tannakiennes, Lect. Notes in Math. 265, Springer, 1972.

[53] A. Scholl Classical motives, Motives (Seattle, 1991), Proc. Sympos. Pure Math., 55, Part 1, Amer. Math. Soc., Providence, RI, 1994, 163-187.

[54] J. P. Serre Letters to Marc Perret and Gilles Lachaud, Oct. 26 and 31, 2000. 
[55] A.N. Shermenev Motif of an Abelian variety, Funckcional. Anal. i Priložen. 8 (1974), no. 1, 55-61. English translation: Functional Anal. Appl. 8 (1974), 47-53.

[56] J. Tate Endomorphisms of abelian varieties over finite fields, Invent. Math. 2 (1966), 134144.

[57] J. Tate Conjectures on algebraic cycles in $\ell$-adic cohomology, Proceedings of Symposia in Pure mathematics 55 (I) (1994), 71-83.

[58] V. Voevodsky A nilpotence theorem for cycles algebraically equivalent to 0, Int. Math. Res. Notices 4 (1995), 1-12.

[59] V. Voevodsky Open problems in the motivic stable homotopy theory, I, in Motives, polylogarithms and Hodge theory, Part I (Irvine, CA, 1998), 3-34, Int. Press Lect. Ser., 3, I, Int. Press, Somerville, MA, 2002.

[SGA7] Groupes de monodromie en géométrie algébrique (SGA7), Vol. II (P. Deligne, N. Katz, éd.), Lect. Notes in Math. 340, Springer, 1973.

\section{Bruno Kahn}

Institut de Mathématiques de Jussieu

175-179 rue du Chevaleret

75013 Paris

France

E-mail:kahn@math.jussieu.fr 